\title{
On the Role of Non-equilibrium Focal Points as Coordination Devices ${ }^{\star}$
}

Antoni Bosch-Domènech, Universitat Pompeu Fabra and CREA, Barcelona, Spain

Nicolaas J. Vriend, Queen Mary, University of London, UK

February 2008

Abstract:

Considering a pure coordination game with a large number of equivalent equilibria, we argue, first, that a focal point that is itself not a Nash equilibrium and is Pareto dominated by all Nash equilibria, may attract the players' choices. Second, we argue that such a non-equilibrium focal point may act as an equilibrium selection device that the players use to coordinate on a closely related small subset of Nash equilibria. We present theoretical as well as experimental support for these two new roles of focal points as coordination devices.

J.E.L. classification codes: C72, C91

Keywords: Coordination game, Focal point, Nash equilibrium, Equilibrium selection, Coordination device.

\footnotetext{
"We thank Irina Cojahurenco and Carlos Trujillo for their help in running and analyzing the experiments, and Edward Cartwright, Herbert Dawid, Dirk Engelmann, Ed Hopkins, Nagore Iriberri, Georg Kirchsteiger, Alan Kirman, Tatjana Kornenko, Marco Mariotti, Axel Ockenfels, Jan Potters, Martin Sefton, Bob Sugden, Nat Wilcox, conference participants of the Game Theory Society (Marseille), Complexity (Aix), FUR (Rome), IAREP/SABE (Paris), ESA (Rome), and seminar participants at Marseille, Queen Mary (London), Pompeu Fabra (Barcelona), Amsterdam, Bielefeld, City (London), Granada, Queen's (Belfast), and UEA (Norwich) for helpful comments and suggestions. Financial help by the Spanish Ministerio de Educación y Ciencia under research project SEJ2005-03891/ECON (AB-D) and by the British Academy through the Small Research Grants Scheme (NJV) are gratefully acknowledged. The usual disclaimers apply.
} 


\section{Introduction}

Many social interactions can be modeled as a coordination game. The players have a common interest, but as there are multiple equilibria, the desired outcomes are only achieved if they mutually adjust their actions in the right way and go for the same equilibrium.

Peyton Young (1993) distinguishes three broad equilibrium selection theories: First, some equilibria may be a priori more reasonable than others (see, e.g., John Harsanyi and Reinhard Selten, 1988). Second, it might be that "agents focus their attention on one equilibrium because it is more prominent or conspicuous than the others" (Peyton Young, 1993, p. 58). Third, expectations may converge on one equilibrium through precedent (see, e.g., Vincent Crawford and Hans Haller, 1990).

Our paper will be most closely related to the second of these theories, but it will differ from all three of them. In our game there will be no equilibrium more reasonable or conspicuous than others, and in the (experimental) setup that we will consider, precedence can be of little help since we will consider a sequence of oneshot games with very limited information feedback.

Thomas Schelling (1960) recognized that in everyday life individuals who are confronted with coordination problems frequently seem to do much better than they would by choosing randomly among the equilibria, and that focal points may play an important role in this by providing a point of convergence for individual expectations. ${ }^{1}$ As Thomas Schelling (1960) put it: "Most situations - perhaps every situation for people who are practiced at this kind of game - provide some clue for coordinating behavior, some focal point for each person's expectation of what the other expects him to expect to be expected to do. Finding the key, or rather finding a key - any key that is mutually recognized as the key becomes the key - may depend on imagination more than on logic; it may depend on analogy, precedent, accidental arrangement, symmetry, aesthetic or geometric configuration, casuistic reasoning, and who the parties are and what they know about each other" (p. 57).

We will consider the role of focal points as a coordination device, but our starting point will be a simple two-player matrix game with common interest and a multiplicity of equivalent and efficient Nash equilibria (NE), none of which stands out. We introduce a salient payoff by reducing one of the equilibrium payoffs, thus eliminating the equilibrium property of the corresponding strategy profile. The question, then, is whether this alteration of the incentive structure (creating a single

\footnotetext{
${ }^{1}$ For a recent discussion of Schelling's analysis, see Robert Sugden and Ignacio Zamarrón (2006).
} 
different payoff) may help individuals to coordinate, even if this payoff is not an equilibrium and it is Pareto dominated by the equilibrium payoffs.

The main contribution of our paper, then, is that we present two new ways in which such a non-equilibrium focal point may help to solve coordination problems. First, we will explain that such a non-equilibrium focal point may be an attractor in itself for the players. Second, we will explain that such a focal point may act as an equilibrium selection device enhancing coordination by becoming a stepping stone from which players jump to a closely related small subset of NE that in themselves are not salient. We will present theoretical as well as experimental support for these two new roles of focal points. The experimental evidence presented will also show that the effect of the focality depends, in a clear way, on the level of the focal payoff, and that the effect of the focal point becomes substantially stronger as the players have time to reconsider and adjust their actions.

The remainder of this paper is organized as follows. In section 2 we present the coordination game that we study, and discuss its relation to the literature. Section 3 derives some theoretical observations, and section 4 explains the design of our experiments. Section 5 proposes a set of hypothesis, while in section 6 we analyze the results of the experiments. Section 7 concludes.

\section{A coordination game and some related literature}

In order to isolate the effect of a salient non-equilibrium payoff, we present a twoplayer artificial game based on the payoff matrix in Figure $1 .^{2}$ The game is represented in normal form with player 1 choosing from the set of rows $\left(r_{1}, \ldots, r_{n}\right)$ and player 2 form the set of columns $\left(c_{1}, \ldots, c_{n}\right)$. To each pair $\left(r_{i}, c_{j}\right)$ corresponds a payoff as indicated in the corresponding cell of Figure 1. The game has thirty equivalent pure NE leading to a payoff of 100 for each player. Any miscoordination leads to a payoff of 0 . Notice that neither risk considerations nor payoff considerations would allow distinguishing any of these NE. Consequently, for this game, in which players are indifferent about the equilibrium they reach, but happy to reach any one of them, and in which there is no communication between the players, it seems exceedingly difficult to say what coordination will be observed and what could explain the choice.

++++ Figure 1 about here ++++

\footnotetext{
${ }^{2}$ Real-life games come with frames. The game is artificial because we try to mute them.
} 
The question we address is whether the introduction of a focal point might help to improve coordination. To this end, we take one of the NE, and replace its payoff $\pi_{N E}$ $=100$ with a payoff $\pi_{F P}$, with $0<\pi_{F P}<\pi_{N E}=100$, whose uniqueness confers salience. Notice that this destroys the equilibrium property of the corresponding strategy profile, as each player could unilaterally deviate to get a payoff of 100 instead, thus realizing one of the NE. Hence, what we get after the manipulation is a game with twenty-nine NE and one salient non-equilibrium payoff forming a focal point. Notice also that the focal point is Pareto dominated by all NE. ${ }^{3}$

We want to stress that this modified payoff would not be salient because of "some kind of conventional priority" (Thomas Schelling, 1960, p. 64) that makes it a special equilibrium. In fact, the focal point that we consider is not an equilibrium at all, and it is prominent only because it has a payoff which is different both from the equilibrium payoffs and from the remaining non-equilibrium payoffs. ${ }^{4}$ That is, the salience is not an extra attribute of one of the equilibria but results from the difference in payoff from the equilibrium payoffs. In this sense our paper belongs to the tradition of formal game-theoretical analysis, as, in our game, subjects will have an opportunity to coordinate thanks to the modification of an element that is structural to the game, a payoff, rather than on contextual cues. ${ }^{5}$ In fact, we strive to make all equilibria "nondescript" (in the sense of Michael Bacharach, 1993) by keeping labels or frames as much indistinct as possible. ${ }^{6}$ In other words, in this game, the players' own description of their options (see D. Gauthier, 1975) should be useless to play the game, except when this description refers to the payoff amounts. ${ }^{7}$

Our purpose in the paper is to analyze how this salient non-equilibrium payoff can become a correlating device that enhances coordination. In particular, we want

\footnotetext{
${ }^{3}$ Hence, the focal point is not "collectively rational" (Robert Sugden, 1995).

${ }^{4} \mathrm{~A}$ focal point, as defined by Schelling, is the Nash equilibrium on which players coordinate. Our focal payoff is not an equilibrium. Only its salience makes it qualify as a "focal point".

${ }^{5}$ With rich framing, how do we adjudicate priority among frames? In the words of Ken Binmore and Larry Samuelson (2006, p. 3), "is an action that is red but not square more focal than an action that is square but not red?". Payoffs, on the contrary, are not subject to such ambiguity.

${ }^{6}$ In pure coordination games with $n$ equivalent equilibria, environmental signals, "labels" or "frames" (see Judith Mehta, Chris Starmer and Robert Sugden, 1994, or Ken Binmore and Larry Samuelson, 2006) become strategically relevant, since they are the only distinct elements that subjects can recognize to coordinate their decisions. In these games, valueless labels become the choice set, and subjects receive utility only if they choose the same label.

${ }^{7}$ If, in addition to the payoff difference, the game contained frames, players might identify more than one salience, making coordination more unlikely and any analysis more difficult. For double focal points, where frame and payoff differences coexist, see Vincent Crawford, Uri Gneezy and Yuval Rottenstreich (2007).
} 
to find out, first, whether and when this different payoff can act as a focal point in achieving coordination upon itself, in preference to the Pareto-dominant equilibrium payoffs. And, second, under what circumstances players use this focal payoff as a device to coordinate on a closely related small subset of NE, even though the equilibria selected are not in themselves salient. We run laboratory experiments under different treatments. Each treatment will be characterized by a different payoff associated with the focal point. And so, we will ask, is the degree of coordination dependent on the focal payoff amount? To state it differently, is there more or less coordination when the amount of the focal payoff approaches the amount of the equilibrium payoffs? And also, how does the coordination shift from the focal payoff to one of the NE when the amounts of the salient payoff change?

The game considered seems to capture an essential feature of many real-life situations, where coordination may be achieved through second-best or dominated outcomes. Consider, for example, a variation of Thomas Schelling's "Meeting in New York City" game. Two people have an appointment at a certain time at a city's main square. As the square is large and crowded, it is imperative that the two people coordinate by choosing the same location. In general, many equilibria will exist. $A$ focal point in the form of a fountain in the middle of the square may be helpful to reach such an equilibrium But both people independently going for the fountain is not necessarily one of the equilibria. Suppose it is raining and the square is bordered by a number of pubs and bars. If one of the people were to go for the focal point of the fountain, the other would be better off heading for the comfort of one of the many bars where he could keep an eye on the fountain while enjoying a drink and avoiding the rain. If both people independently headed straight for one of the bars, miscoordination would be likely, unless they happened to choose exactly the same bar.

Focal points may play a similar role with respect to contributions in threshold public good games, in which some valuable public good will be realized if and only if a certain threshold in the number of contributions is reached, and in which further contributions beyond the threshold do not further enhance the public good (see, e.g., Theo Offermans, 1997). In such games, typically many asymmetric equilibria exist, equilibria in which exactly the required threshold is reached, with some people contributing and others not. Such asymmetric equilibria may be difficult to realize. And the symmetric equilibrium of nobody contributing might prove more attractive over time than any of the asymmetric equilibria in which the public good is realized (see John Ledyard, 1995). Now, let us assume that policy makers do not care which 
of the equilibria is realized as long as the outcome is not the symmetric nocontributions NE. This is, again, an equilibrium selection problem for which a nonequilibrium focal point may help. Suppose policy makers introduce a law making contributions compulsory for everybody. As the realization of the public good requires only a certain threshold of contributions, everybody contributing does not constitute a NE. But such a law might very well create a focal point (see Richard McAdams, 2000) that could help to solve the equilibrium selection problem by eliminating the symmetric no-contributions NE, thus reaching the threshold of contributions while tolerating some free-riding. ${ }^{8}$

This paper is related to a strand of literature concerned with repeated coordination games. In these, it is the repetition of the game with the same players -its history, so to speak- that allows some equilibria to become focal points on which players can learn to coordinate (see Vincent Crawford and Hans Haller, 1990, or Andreas Blume and Uri Gneezy, 2000). Instead, we consider a situation in which players play the game repeatedly but are matched randomly. While the game has a history for each player, this history does not make some equilibria focal. It is a weak history that does not de-symmetrize the game. To help coordination, an asymmetry is exogenously created in the payoffs. Unlike Vincent Crawford and Nagore Iriberri (2007b), where their game (with frames) is analyzed as if the payoffs were perturbed to accommodate the instinctive attraction of salience, in our game (where frames should not exist), a payoff is actually perturbed (shaved) in order to give it salience, albeit simultaneously depriving it of its equilibrium properties: While positive salience (attraction) is conferred to one payoff by distinguishing it form the rest, negative salience (repulsion) is simultaneously conferred to it by shaving its value. ${ }^{9}$ In the agents' minds, both types of salience will fight for prevalence.

Our paper also relates to previous research on the powerful effects of nonequilibria. The phenomenon that players may be attracted to non-equilibria or that out-of-equilibrium payoffs may have an impact on the equilibrium towards which

\footnotetext{
${ }^{8}$ Why not make one of the asymmetric equilibria a focal point through some expressive law? There may be so many possible equilibria that it may be difficult to decide which one to make focal by law. Asymmetric equilibria in public goods games might be politically unattractive, and a symmetric focal point might be easier to sell as it is not only inherently more conspicuous but probably also seen as more fair.

9 From an implementation perspective, subtracting from a payoff may be a feasible procedure, while adding to a payoff may not be. In any case, making a payoff salient by increasing it would make the problem of coordination trivial, from a behavioral perspective: The players "should not have any trouble in coordinating their expectations" (John Harsanyi and Reinhard Selten, 1988) p. 81) at the single payoff dominant equilibrium.
} 
players are attracted has been pointed out, e.g., by Thomas Schelling (1960), Margaret Gilbert (1989), Russel Cooper, Douglas DeJong, Robert Forsythe and Thomas Ross (1990), and Giovanna Devetag (2000), who all explain this phenomenon either by risk or fairness considerations. Our game is different in that, instead of risk or fairness considerations, the importance of the out-of-equilibrium payoff is simply due to its salience.

Finally, Joel Huber, John Payne and Christopher Puto (1982) present a phenomenon nowadays well-known in marketing that seems very similar in spirit to the role of our focal points. Suppose consumers want to compare various products across a number of dimensions (size, quality, color, price), with different dimensions favoring different products. This might be a difficult consumer problem. They found that the following has a serious impact on consumer decisions: introducing an asymmetrically dominated alternative. That is, they introduce a new commodity in the market that is dominated in all dimensions by one of the existing products. The result is that this dominating product is going to be hugely favored by the consumers. This is similar in spirit to the use of the focal point in our game. In both cases individuals face a difficult decision problem full of uncertainty. In the marketing case this uncertainty is due to the difficulty of comparing products across many dimensions. In our case it is strategic uncertainty concerning the choices made by other players. In both cases an option is introduced that as such may perhaps not be a solution. But this option may act as a stepping stone to reach such a solution.

\section{Experimental design}

All sessions took place in the computerized experimental laboratory (LeeX) at the Universitat Pompeu Fabra in Barcelona between 2002 and 2004, and lasted about one hour each. The subjects were volunteer undergraduate students in Humanities, Commercial Studies, Business Administration, Law, Economics and Political Science. In each session there were 18 players simultaneously in the laboratory. Participants sat in front of personal computers, and could not observe the screens of other players nor communicate with them. They were given written instructions (see Appendix 2). The instructions contained information about the mechanics of the session and about the subjects' gains, which consisted of a payoff that depended on their decisions and the decisions of the subjects they were matched with. On average, subjects made about $€ 12$ in addition to the show-up fee of $€ 3$. 
Each session included two experiments (a one-shot version and a 50-period version), which were carried out sequentially. ${ }^{10}$ We choose to experiment both with a one-shot game and with a repeated game with random matching. One-shot games allow concentrating on the role of focal points. But in games with some complexity, subjects may need more that one round to observe the focal point, and more than one round to learn to coordinate. We choose a random matching scheme in every round to abstract from any reputation or signaling effects. Such a random matching scheme allows subjects to acquire experience while avoiding creating a supergame. The basis for both experiments is the game with a large number of equivalent $\mathrm{NE}$ presented in Figure 1. To help avoid any of these NE being more prominent (as this would imply the occurrence of spurious focal points), we added a row and a column with only 0 payoffs at each side of the matrix. ${ }^{11}$ The largely empty center of the matrix is intended to serve the same purpose. Notice also that no equilibria lie on the diagonal, and that there are no labels distinguishing the actions. ${ }^{12}$ We used a number of variations of this game that consisted of rotating or mirroring the payoff matrix and changing the colors assigned to the row and column players. The instructions of the experiments as well as some sample screenshots can be found in Appendix $2 .{ }^{13}$

We shaved one of the 100 payoffs to make it focal and used this focal payoff as a treatment variable to create four different treatments for the two experiments in each session. The values for the focal payoffs used were 46, 87 and 99 points, respectively. ${ }^{14}$ In addition, we ran a control treatment in which this payoff was 100 points, effectively eliminating the focal point. ${ }^{15}$ This control treatment was meant to alert us of the existence of any nuisance attributes on which subjects could coordinate. Apart from the focal payoffs there was no difference between the treatments. We ran eight sessions with the "46" treatment, and six sessions with each of the treatments " 87 ", "99" and " 100 ".

\footnotetext{
${ }^{10}$ Due to a computer glitch, one session (46.2) lasted only 45 periods. All analyses have been adjusted for this. For example, when considering the last ten periods we use periods 36-45 rather than periods 41-50 for this session.

${ }^{11}$ Notice that this implies that each of the four corners of the matrix constitutes a Nash equilibrium as well, with payoffs as low as those of the worst possible miscoordination. We will neglect these four non-strict Nash equilibria throughout the paper.

12 Once the instructions were read, subjects faced the payoff matrix on the screen for the remaining of the session. No natural language was used on the screen, thus limiting its possible confounding effect (see, e.g., Glenn Harrison, 2005).

${ }^{13}$ These instructions were translated into Spanish for the experiments. The complete set of matrices used is available from the authors.

${ }^{14}$ In one session (46.4), by mistake we actually used a focal payoff of 47 rather than 46 .

15 The monetary reward in Euros per experimental payoff point was constant across the treatments. Notice that making the focal payoff equal to 100 takes the game back to its original format as described in Figure 1.
} 
At the start of each session, the subjects were split into two separate groups: row and column players. Each group was assigned a color that was used in the matrix on the screen. For example, 'yellow' players choose an action by clicking one of the yellow cells on top of the columns, while 'blue' players did this by clicking on one of the blue cells preceding the rows. The colors used changed from session to session.

All subjects had to play in both of the two experiments of each session. In the first experiment subjects played, with no time restriction, a one-shot version of the game by simply choosing one row (column). This was for a high payoff of $€ 1.00$ per 10 experimental payoff points. Once their decision was made, they did not receive any feedback on the results of the game, and proceeded straight to the second experiment. This second experiment used exactly the same payoff matrix, and each player kept the same role (either row or column player). In this second experiment the subjects would play 50 rounds of the same game, randomly re-matched in each round to one of the players in the other group. In this experiment, after each round each player was told his payoff, but not the action chosen by the other player The monetary reward in the 50-period second experiment was $€ 0.05$ per 10 experimental payoff points.

The purpose of the first experiment was to test whether introspection, logic and imagination would suffice for the players to coordinate successfully. ${ }^{16}$ Moreover, the first experiment prepares the stage for the second one. Paying up to $€ 10.00$ for a single decision in a two-player coordination game, and giving the players all the time they wanted, was done to motivate and allow the players to examine the characteristics of the game carefully right at the beginning of the first experiment, thus avoiding a slow and leisurely start of the second experiment (with agents learning and discovering the game 'on the fly'). Notice that since we did not provide any feedback on the first experiment, there is no endowment or learning effect related to the outcomes of the first experiment.

\section{Theory of focal points}

The existing literature on the role of focal points consists essentially of finding ways to formalize a justification to coordinate on a focal equilibrium. When there are multiple equilibria, the focal point can act as an equilibrium selection device in the sense of rendering one of the equilibria more prominent than the other(s). This idea

\footnotetext{
${ }^{16}$ Schelling (1960): "But in the final analysis we are dealing with imagination as much as with logic" p. 58).
} 
of players choosing one equilibrium that stands out has been formalized in various ways (see, e.g., Vincent Crawford and Haller, 1990, Robert Sugden, 1995, Michael Bacharach and Michele Bernasconi, 1997, Maarten Janssen, 2001, and André Casajus, 2001).

In this section we show that this literature can also help to elucidate that a focal point may play an important role in achieving coordination, even when this focal point is not itself an equilibrium. As we will show, this theory explains both of the two roles of a focal point that we present in this paper, although neither role of a nonequilibrium focal point has been recognized in the literature.

Consider, as an illustration, the following two-player simultaneous-move coordination game. Suppose there are five white balls on a table, and if both players choose the same ball, they get a payoff of 100 , whereas they get nothing in case they do not coordinate. This game has five Nash equilibria in pure strategies. Now, suppose one of the five balls is red, while the rest of the game is as above. This game still has the same Nash equilibria, but one of them is more prominent than the others. Next, suppose that the payoff for both players choosing the red ball is 80 while the rest again stays as above. This allows for a partial Pareto ranking of the equilibria. The red equilibrium is Pareto-dominated by any of the white equilibria, but it is still the most prominent equilibrium.

Bacharach and Bernasconi (1997) showed formally how the most focal of the $\mathrm{NE}$ can be selected as the unique solution of this game. ${ }^{17}$ The idea is the following. Players do not choose actions as such, but instead classify actions into options (partitioning the strategy set), and then choose an option. What is an option depends on what actions the players can distinguish, given the frame in which they perceive the game. In the present game, it seems likely that players distinguish only two options: choosing a red ball or choosing a white ball. If a player opts for choosing a 'white ball', then, following the Principle of Insufficient Reason, she randomly picks any of the white balls. In addition, a player could choose any ball without distinguishing any characteristic. This means that the underlying game, for which the payoff matrix is shown in Figure 2, is transformed into a different description as shown in Figure 3, where the payoffs are the expected payoffs. Thus, for example, the expected payoff if both players choose 'white ball' is 25 , as there is only a 1 in 4 chance of both players choosing exactly the same white ball leading to a payoff of 100. As we see, in the base game in Figure 2 there are five NE (shaded): four equivalent NE and one Pareto dominated equilibrium involving the focal point. In the

\footnotetext{
${ }^{17}$ See also Bacharach (2006).
} 
transformed game in Figure 3 we have two NE: one in which both subjects choose 'white', and one in which both players choose 'red', which is now the unique Pareto superior NE, and, in Bacharach and Bernasconi's terminology, the unique Variable Frame Theory (VFT) solution of the game. In other words, VFT formalizes the fact that one NE is more focal than other NE, and transforms it into the unique Pareto superior NE.

++++ Figures 2 and 3 about here ++++

We now apply the same principles to our coordination game. Suppose that one of the 100 payoffs is replaced by an 87 payoff. Following the previous argument, this game can be transformed into the description of the game presented in Figure 4 (Description 1), in which we distinguish three options: choose a row (column) with the focal point, choose any of the other rows (columns) that include two NE, or choose just any of the rows (columns). Figure 4 shows two NE in the transformed game, and the one involving the focal point is again the superior one. But there is a noteworthy new element here. The focal point was not a NE in the underlying game, yet it emerges as the unique Pareto superior NE when looked at from the perspective of VFT.

++++ Figures 4 and 5 about here ++++

However, if we return to the underlying game, we see that if the players are going for this solution, it is profitable for each player to deviate unilaterally to the Nash equilibrium that shares the same row (column) with the focal point. We will call such a Nash equilibrium (in which one player goes for the focal point while the other chooses a best-reply to this) an Associated Nash Equilibrium (ANE). Therefore, one can reach another description of the game following a VFT transformation of the underlying game. That is, the option 'row with $2 \mathrm{NE}$ ' can be split into 'associated row with 2 NE' and 'other row with 2 NE'. This leads to Description 2 in Figure 5, in which we distinguish two types of NE. First, there is a NE with both players going for any of the other NE. But there are also two additional NE. The superior NE of both players going for the focal point is replaced by two equilibria with one player sticking to the focal point while the other deviates to the ANE. These two ANE are superior to the 'other' NE. By this procedure, we have formalized the equilibrium selection, since two of the twenty-nine NE of the underlying game (i.e., the two ANE) are singled out as 
the possible solutions for this game. The application of the VFT principles can be summarized as follows:

Theoretical observation 1: The principles of VFT imply that a non-equilibrium focal point can become the unique Pareto superior Nash equilibrium.

Theoretical observation 2: The principles of VFT imply that a non-equilibrium focal point can transform a small subset of NE, the ANE, into Pareto superior Nash equilibria.

And now, we can face the question of what are the participants' choices in the coordination game. Are they enticed by the equilibria in the initial description of the game (Description 0) as shown in Figure 1 with one 100 payoff replaced by a "treatment" payoff, e.g., 87, or by one of the equilibria in Descriptions 1 and 2, in Figures 4 and 5 ? The question is an empirical one that, as we will see in the next section, depends in part on the relative payoffs involved. To help understand how this can be, we classify the participants into levels, according to the depth of their descriptive analysis and their resulting beliefs on other players' levels. ${ }^{18}$

Level-0 players act on Description-0 (or initial) and best-reply to their beliefs that all other subjects act best-replying to their beliefs that all others act on the only description available, or Description-0.

Level-1 players act on Description-1 of the game (Figure 4) and best-reply to their beliefs that all other players are either Level-0 or Level-1 in some proportion $p$. Accordingly, they will try to reach (depending on $p$ ) either the equilibrium with an 87 payoff or the one with a 13.79 payoff.

Level-2 players act on Description-2 of the game (Figure 5). But what happens if a Level-2 player believes that a sufficient large number of players have also reached his description level? If she moves to the ANE and the matched player also moves to his ANE, then their payoff will be zero (second row and second file in

\footnotetext{
18 This classification is reminiscent of the levels-of-reasoning models (Rosemarie Nagel, 1995, Dale Stahl and Paul Wilson, 1995, Antoni Bosch-Domènech, José Garcia-Montalvo, Rosemarie Nagel and Albert Satorra, 2002, Colin Camerer, Teck-Hua Ho and Juin-Kuan Chong, 2004, Vincent Crawford and Nagore Iriberri, 2007a), with a different variable frame description being attained at each level of reasoning. Yet, in the present description, players believe that a proportion of other players are at their own level of reasoning. By the way, sometimes VFT and levels of reasoning are considered alternative theories to explain observed coordination results (see Nicholas Bardsley, Judith Mehta, Chris Starmer and Robert Sugden, 2006). But they need not be. In our game, considering a cognitive hierarchy of VFT descriptions may help to understand the experimental results.
} 
Figure 5). For their payoffs to be 100 , only one of the two has to move to the ANE. In other words, one of the two has to best-reply as if a large number of the other players were at Level-2, but the other player has to best-reply as if others were at Level-1. ${ }^{19}$

Clearly, the players' depth of analysis may vary as they keep playing and learning the game. Consequently, it would not be surprising if a player who starts as a Level-0 player would end the game as a Level-2 player.

\section{Hypotheses}

On the basis of the analyses of the previous section, we state the following hypotheses.

\section{Hypothesis 1:}

The underlying game we presented in Figure 1 without the focal payoff (treatment "100") does not contain any spurious focal points facilitating successful coordination, and hence it presents the players with a non-trivial coordination problem.

But when we introduce a non-equilibrium Focal Point (FP) to the underlying coordination game by shaving one of the 100 payoffs, this will substantially enhance coordination success. Therefore:

\section{Hypothesis 2:}

In the 46, 87 and 99 treatments we will observe more coordination success than in the 100 treatment.

As the attraction of the FP as well as the temptation to deviate from it may depend on the amount of the focal payoff:

\section{Hypothesis 2b:}

The larger the focal payoff, the higher the coordination success.

\section{Hypothesis 3:}

The coordination enhancement in the focal treatments mentioned in hypothesis 2 is achieved by coordinating either on the FP (even though not an equilibrium itself) or on any of the ANE, and not by improved coordination on the other NE.

\footnotetext{
${ }^{19}$ Here we have to depart from Bacharach's theory, where two players who reach the same description will choose the same strategy. But his theory of team reasoning could be relevant with Description-2 of the game, when payoff maximization requires that the matched subjects do not choose the same strategy.
} 


\section{Hypothesis 3b:}

The larger the focal payoff, the more subjects make use of the FP (choosing either the focal or associated action), and the greater the role played by the FP relative to the ANE.

As argued above, the FP is not an equilibrium, and each player could deviate unilaterally to one of the ANE. However, if both column and row players were to make such a move, miscoordination with a payoff of zero would result. Therefore:

\section{Hypothesis 4:}

In each session, to the extent that the ANE are used successfully to coordinate, the relative shares of coordination success of ANE1 and ANE2 will not be 50-50, but one of the two will become dominant.

The experimental evidence that follows will help to substantiate or dismiss these hypotheses.

\section{Experimental results}

In this section we present the substance of the experimental evidence supporting the two new roles of focal points, quantifying their effect, and thus providing a test of the hypotheses discussed above. For a detailed analysis of the experiment we refer the reader to Appendix 1, where a number of experimental observations are described and statistically tested. The references below to the numbered observations refer to these observations in Appendix 1.

A first summary of the results appear in Table 1, which presents the average earnings (excluding the show-up fee of $€ 3.00$ ) per player for each treatment in both experiments combined (one-shot plus 50 periods). In broad terms, Table 1 shows that coordination is enhanced by the introduction of a non-equilibrium focal point, and that it increases with the amount of the focal payoff. We now turn to a detailed analysis of the experimental results.

++++ Table 1 about here ++++

Hypothesis 1, that the initial game (Figure 1) posed a non-trivial coordination problem is largely confirmed. Figure 6 shows, for each of the four treatments, the average number of times that players choose the focal action, the associated action, and the 
most frequently chosen other (i.e., not focal or associated) action in the one-shot game. 2021

++++ Figure 6 about here ++++

In the "100" treatment (see Figure 6, last entry) there was not one action that stood out (Obs. 1), and the coordination success was not higher than what would be expected from random behavior (Obs. 5).

++++ Figures 7 and 8 about here ++++

Similarly, as illustrated in Figure 7, which shows the average frequency of the most chosen action (summed over row and column players) in each period in the "100" benchmark treatment, in the 50-period game of the "100" treatment the most frequently chosen action was selected slightly more than would be implied by random choice (Obs. 13), but this frequency did not increase over time (Obs. 14).

In Figure 8, which shows the average expected payoffs in the 50 periods of the "100" treatment, we observe that they increase somewhat over time (Obis 16), and become higher than would be expected from random behavior (Obs. 15), but not substantially so (Obs. 17). That there is no spurious focal point was confirmed in the focal treatments ("46", "87", "99"), as neither in the one-shot game nor in the 50period game (see Figure 9, which shows the 50-period actions in all three focal treatments) is there an "other action" (apart from the focal and associated actions) that stands out (Obs. 2 and 18), and the frequency of the most chosen other action does not increase over time (Obs. 19).

++++ Figures 9 and 10 about here ++++

${ }^{20}$ Several one-shot experiments have shown that participants "use subtle features of the experimental environment to solve the problem of coordinating on a common mode of reasoning" (Nicholas Bardsley, Judith Mehta, Chris Starmer and Robert Sugden (2006) p. 34). Not so in our experiment. Perhaps because we have made an effort to eliminate any "labels" that could suggest "precedence", "analogy", "geometric configuration" or any of the rules that subjects may apply to coordinate. And, from oral debriefing, we know that even when they are aware of the focal point, the pull of a salient payoff apparently was often not enough to overcome the push of a lower payoff, at least before a process of trial and error leads them to coordinate.

${ }^{21}$ To find the most frequent action we take the sum of the most frequently chosen column and the most frequently chosen row. 
Hypothesis 2, that the focal point would substantially enhance coordination success, is fully confirmed. Comparing Figures 8 and 10, which show the expected average payoffs in the four treatments, we observe that the expected payoffs in the three focal treatments were significantly above those in the non-focal treatment (Obs. 6 and 24), and these expected payoffs were increasing over time (Obs. 25). As a result, the expected payoffs in periods 41 to 50 were 1.8 times higher in the "46" treatment than in the "100" treatment, 3.1 times higher in the "87" treatment, and 3.5 times higher in the "99" treatment. 22

Hypothesis $2 b$, that the coordination success would increase with the amount of the focal payoff was largely confirmed. On average this was correct in both the one-shot game (see Table 3) and the 50-period game (see Table 6 and Figure 10), but some of the differences were not significant (Obs. 11 and 37).

Hypothesis 3, that the coordination enhancement in the focal treatments is due to the focal point is essentially confirmed. The expected payoffs realized through any of the other NE are not higher in the focal treatments than in the non-focal "100" treatment in the one-shot as well as the 50-period game (Obs. 7 and 26 and Figures 8 and 10), and these expected payoffs do not increase over time (Obs. 27). As illustrated in Figures 6 and 9 , in each of the focal treatments subjects make use of the FP (choosing either the focal or associated action) more than what should be expected from random behavior, in the one-shot as well as the 50-period game (Obs. 3 and 20), and the frequency is increasing over time (Obs. 21). The expected payoffs realized through the FP are not higher than those coming through the best of the other NE in the one-shot game (Obs. 8), while they tend to be higher in the 50-period game (Obs. 28). The same applies when comparing the coordination success of the best of the ANE with the best of the other NE. In the one-shot game there is not much of a difference (Obs. 9), but the best ANE tends to be more important in the 50period game (Obs. 29). The resulting expected payoffs realized through either the FP or the ANE tend to be higher than those coming through the other NE combined in the one-shot game (Obs. 10) and the 50-period game (Obs. 30). The expected payoffs due to the FP as well as the ANE increase over time (Obs. 31, 32, and 33).

\footnotetext{
${ }^{22}$ Yet, we also observe that some subjects who spot and play the FP or ANE become discouraged after a while if they do not find the appropriate responses of their matches. Observing and using the focal cues may not be evolutionary successful in an environment where many participants do not respond to the focal cues. This observation is reminiscent of the Ken Binmore and Larry Samuelson (2006) theoretical conclusion that "increased monitoring that would be efficient if performed by the whole population need not enjoy evolutionary success" (p. 40).
} 
Hypothesis $3 b$, that the FP is more frequently used the larger the focal payoff, and that the FP itself becomes more important relative to the ANE the larger the focal payoff, are confirmed. Both in the one-shot game and the 50-period game (see Figures 6 and 9), subjects tend to use the FP more (choosing either the focal or associated action) the larger the focal payoff (Obs. 4 and 23). In the one-shot game, there is no significant difference in the importance of the FP relative to the ANE (Obs. 12). But in the 50-period game, the importance of the ANE increases over time in the "46" and "87" treatments (Obs. 34), and related to this, the larger the focal payoff, the more important the FP becomes relative to the ANE (Obs. 22 and 38).

++++ Figure 11 about here ++++

Hypothesis 4, that, to the extent that the ANE are used successfully to coordinate, one of the two ANE will become dominant is confirmed on average, although statistically this does not appear substantial (Obs. 35). Figure 11 shows the average relative share in the expected payoffs of each of the ANE for each treatment (see also Figure 10). As we see, in all focal treatments one ANE ends up being chosen much more often than the other. ${ }^{23}$ Of the expected payoffs realized through the two ANE in periods 41 to 50 , the share attributed to the first ANE is on average 83, 89, and $87 \%$ for the "46", "87" and "99" treatments respectively. The average shares realized through the first ANE increase from 51 to $83 \%$, from 72 to $89 \%$, and from 86 to $87 \%$ in the "46", "87" and "99" treatments respectively, although statistically the increase is only significant in the "87" treatment (Obs. 36). In other words, on average it seems that row players learn not to deviate from the FP when column players tend to deviate, and vice versa.

\section{Concluding remarks}

"When people are otherwise at a loss for how to coordinate, it takes surprisingly little to guide expectations and behavior" (Richard McAdams (2000) p. 1652). In the paper we consider a coordination game with a large number of equivalent NE, where players have a difficult time coordinating. But when one of the equilibrium payoffs is made salient by shaving its value, thus making it loose its equilibrium properties, it nevertheless appears to induce coordination. And the degree of coordination

${ }^{23}$ That groups of experimental subjects end up in one specific focal NE for no particular reason was also observed by Ken Binmore, Joe Swierzbinski, Steven Hsu and Chris Proulx (1993). 
depends inversely on the difference in value between the focal payoff and the equilibrium payoffs: less is more, that is, less shaving is more coordination. ${ }^{24}$

The following should be emphasized:

1. Focality in the experiments is not conferred by labeling or any other "conventional priority", but by shaving a payoff.

2. The salient payoff is not an equilibrium.

3. Variable Frame Theory together with a levels-of-reasoning approach are helpful in understanding the observed outcomes.

4. Focality plays an important role, but players do not come to it simply through introspection or precedent. Practice and learning are crucial.

5. In our game, where all NE are undistinguishable, the smallest shaving of a single payoff achieves the highest degree of coordination.

6. Players learn fairly quickly to discriminate between the two similar ANE.

7. The analysis in the paper is static. How subjects adapt and learn should be the subject of another paper.

${ }^{24}$ Perhaps Schelling's example of the painted line on the road is a good description of what is happening. Drivers have reasons to obey the line even if the state does not provide enforcement of it. But if the state draws the line too far over, drivers will ignore the line. One could say that the probability that a certain convention helps coordination increases with "the strength of its case", or, in our terms, the amount of the focal point's payoff. 


\section{Appendix 1: Analysis of the experimental results}

\section{First experiment: One-shot}

The "100" treatment is a benchmark treatment, identical to the original game in Figure 1. The results of the treatment tell us how difficult the coordination task is, and whether there appears to be any spurious focality in the matrix. On average, in the "100" treatment, the number of times the most chosen action was selected is 4.3 (see Figure 6). This is lower than the maximum to be expected if the players choose uniform randomly among the available actions, which is 4.5 (2.25 row plus 2.25 column players). ${ }^{25}$ Hence, there appears to be little, if any, spurious focality attracting the players' choices.

++++ Table 2 about here ++++

Experimental observation 1 (one-shot): In the "100" treatment, we cannot reject the null hypothesis that the median of the most frequently chosen action equals the theoretical value implied by random choices. ${ }^{26}$

This is confirmed by the average number of times the most chosen other action was selected in the "46", "87" and "99" treatments: 5.0, 4.2, and 3.7 respectively. ${ }^{27}$ See Table 2 and Figure 6. In other words, apart from the focal and associated actions (to which we turn below), it is not the case that there is a single row and column that stand out more than what would be expected for uniform random behavior.

Experimental observation 2 (one-shot): In each of the focal ("46", "87" and "99") treatments, we cannot reject the null hypothesis that the median of the most frequently chosen other action equals the theoretical value implied by random choices. ${ }^{28}$

Aggregating focal and associated choices, the average number of times that the subjects made use of the FP is $8.2(46 \%)$ in the "99" treatment. This number falls to $6.0(33 \%)$ in the

\footnotetext{
${ }^{25}$ We approximated this expected maximum numerically, simulating the uniformly random choice of players over rows and columns, and computing the maximum for one million runs. ${ }^{26}$ One-tailed binomial test. Here, as for almost all tests, we use the sessions as independent observations. We will indicate explicitly any deviation from this.

${ }_{27}$ Uniform random behavior would have led to an expected maximum of 4.3 in these treatments. This is lower than for the "100" treatment, as the maximum is taken ignoring the numbers for the focal and associated actions.

${ }^{28}$ One-tailed binomial test, using the individual actions as independent observations.
} 
" 87 " treatment, and to $4.5(25 \%)$ in the " 46 " treatment. ${ }^{29}$ In other words, it appears that the players are attracted by either the FP or the ANE.

Experimental observation 3 (one-shot): In each of the focal ("46", "87" and "99") treatments, subjects make use of the FP (choosing either the focal or associated action) significantly more than what should be expected from uniform random behavior (i.e., 2.4). ${ }^{30} 31$

What is more, as can be seen in Table 2, the closer the focal payoff is to the NE payoffs, the higher the average number of times both the focal action and the associated action are chosen.

Experimental observation 4 (one-shot): In the "99" treatment, subjects make use of the FP (choosing either the focal or associated action) significantly more than in the "87" or "46" treatment, ${ }^{32}$ while there is no significant difference between the " 87 " and "46" treatments. ${ }^{33}$

What do these observed choices imply in terms of expected coordination success? The measure of coordination success we use is the expected payoffs, taking into account the actions of all players in a session and the fact that each given row (column) player is equally likely to be matched to any of the column (row) players in that session. ${ }^{34}$

++++ Table 3 about here ++++

In Table 3, we present the expected payoffs for the individual sessions for each treatment and how these are attributed to the FP, the two ANE, and the other NE. ${ }^{35}$ As we see, there is

${ }^{29}$ Two outliers seem noteworthy in Sessions 9 and 18, where the focal action was chosen by 10 and 11 subjects. This appears to be a chance effect. It "reverted to the mean" as subjects went into the second experiment, as can be seen from Table 5 below.

${ }^{30}$ Since 18 players per session choose among 15 actions (ignoring the empty rows and columns), uniform random behavior would lead to an expected frequency of 1.2 players choosing any specific action.

${ }^{31}$ One-tailed binomial test based on individual actions, significant at any conventional level for each treatment.

${ }^{32}$ One-tailed $\chi^{2}$ test based on individual actions, significant at 10 and $1 \%$ respectively.

${ }^{33}$ Essentially the same applies to the focal action as such (with the significance levels at 10 and $2 \%$ respectively), while there is no significant difference between the treatments as far as the associated actions are concerned.

${ }^{34}$ We use expected payoffs rather than actual observed payoffs, because the latter measure is sensitive to the actual realization of the random matching. Our measure essentially corresponds to the coordination index used in Nicholas Bardsley, Judith Mehta, Chris Starmer and Robert Sugden (2006).

${ }^{35}$ We distinguish the first and second ANE as follows. For each individual session we arbitrarily label the ANE that leads to the highest expected payoffs as "first", and the other as "second". We do the same, but independently, for the 50-period experiment. Notice that this 
some variation among the sessions, but not that much. The main exception is session 99.3 in the "99" treatment. Whereas the average expected payoff for all other sessions in all treatments is 17 , with a minimum of 7 and a maximum of 27 , the outlying session 99.3 has an expected payoff of 77 . If players choose their actions uniform randomly, the expected payoff per player would be just over 13 (depending on the focal payoff, it ranges from 13.1 in the "46" treatment to 13.3 in the "100" treatment). In the "100" treatment, the actual expected payoff is only slightly higher, namely 14.2. Thus without a focal point there is little coordination success, and there appears to be no spurious focality, due to nuisance attributes, facilitating coordination.

Experimental observation 5 (one-shot): In the "100" treatment, the expected payoffs are not higher than would be expected from uniform random behavior. ${ }^{36}$

In the focal treatments, we find somewhat higher expected payoffs than in the "100" treatment, but in the "46" and "87" treatments this is only marginally so: 16.9, 16.5 and 29.1 for the "46", "87" and "99" treatments respectively.

Experimental observation 6 (one-shot): The expected payoffs in the treatments with focal point are significantly higher than in the "100" treatment. ${ }^{37}$

The expected payoffs realized through the other NE are 11.4, 7.2 and 6.0 in the "46", "87" and "99" treatments respectively, whereas it was 14.2 in the "100" treatment. This implies that the higher expected payoffs in the focal treatments seem due to the attractiveness of the FP and ANE as such, and not to improved coordination on the other NE.

Experimental observation 7 (one-shot): The expected payoffs of the other NE in the focal treatments are not higher than in the "100" treatment. ${ }^{38}$

The expected payoffs realized through either the FP or the ANE is 5.5, 9.3 and 23.15 for the "46", "87" and "99" treatments respectively. We now compare this with the expected payoffs realized through the other $\mathrm{NE}$.

implies that these labels do not necessarily refer to the same ANE in the one-shot and the 50-period experiment.

${ }^{36}$ One-tailed binomial test.

${ }^{37}$ One-tailed robust rank-order test, significant at $10 \%, 1 \%$ and $5 \%$ for the "46", "87", and "99" treatments respectively.

${ }^{38}$ One-tailed robust rank-order test. In fact, in the "87" and "99" treatments the expected payoff realized through the other NE is significantly lower than in the "100" treatment (both at $1 \%$ ). Similar observations can be made if we average over all the (other) NE. 
Experimental observation 8 (one-shot): In none of the focal treatments is the expected payoffs realized through the FP higher than those coming through the best of the other NE. ${ }^{39}$

Experimental observation 9 (one-shot): In the "99" treatment, the expected payoffs realized through the best of the ANE are higher than those coming through the best of the other $N E,{ }^{40}$ while there is no significant difference in the "46" and " 87 " treatments.

Experimental observation 10 (one-shot): In the "99" treatment, the expected payoffs realized through either the FP or the ANE are higher than those coming through the other $\mathrm{NE},{ }^{41}$ while there is no significant difference in the "46" and " 87 " treatments.

Experimental observation 11 (one-shot): The expected payoffs are higher in the "99" treatment than in the "87" treatment and than in the "46" treatment, ${ }^{42}$ while there is no significant difference between the " 87 " and the " 46 " treatments.

Next, we consider the importance of the FP relative to the ANE in the various treatments. The average expected payoffs realized through the FP as share of those realized through either the FP or the ANE are 34, 42, and 44\% for the "46", "87" and "99" treatments respectively.

Experimental observation 12 (one-shot): There are no significant differences between the "46", "87" and "99" treatments in the payoffs realized through the FP relative to those realized through the ANE. ${ }^{43}$

\section{Second experiment: 50 rounds}

We now move on to the second experiment, in which the same subjects played 50 rounds of exactly the same game as in the one-shot experiment, with random matching in each period.

We first turn to the "100" benchmark treatment, and look at the average frequency of the most chosen action (summed over row and column players) in each period, as shown in Figure 7. Table 4 gives the frequencies of the individual "100" sessions. We observe that the average frequency (4.9 for periods 1-50) stays just above the expected value in case of random selection of actions, namely 4.5 . What is more, there is little variation in the average frequency during the 50 periods, with the average increasing from 4.7 in periods $1-10$ to just 5.2 in periods $41-50$.

\footnotetext{
${ }^{39}$ Wilcoxon signed ranks test.

${ }^{40}$ Wilcoxon signed ranks test, significant at $6.3 \%$.

${ }^{41}$ Wilcoxon signed ranks test, significant at $7.8 \%$.

${ }^{42}$ One-tailed robust rank-order test, significant at 5 and $10 \%$ respectively.

${ }^{43}$ One-tailed robust rank-order test.
} 
Experimental observation 13 (50 periods): In the "100" treatment, the most frequently chosen action is selected more than what would be implied by random choices. ${ }^{44}$

Experimental observation 14 (50 periods): In the "100" treatment, the most frequently chosen action does not increase over time. ${ }^{45}$

That the coordination problem without a focal point is a difficult one, and that the 50 periods do not help a great deal in converging on one row/column is confirmed by the fact that the degree of success in achieving coordination hardly improves over time in the "100" treatment. The average expected payoff per player stays well below 20 (see Figure 8), increasing from 13.42 in periods $1-10$ to 18.09 in periods 41-50 (see Table 4). As Table 4 also shows, there are only two sessions where the average expected payoff in periods 41-50 exceeds 20 , with the highest value being only 24.8 .

++++ Table 4 about here ++++

Experimental observation 15 (50 periods): In the "100" treatment, the expected payoffs become higher than what would be expected from random behavior. ${ }^{46}$

Experimental observation 16 (50 periods): In the "100" treatment, the expected payoffs increase somewhat over time. ${ }^{47}$

Experimental observation 17 (50 periods): In the "100" treatment, the one-sided 95\% confidence interval for the median value of the expected payoffs in periods 41-50 ranges from 0 up to (but not incl.) 24.82. ${ }^{48}$

Thus, although in the non-focal "100" treatment the subjects somewhat succeed in singling out one action to achieve coordination, the effect does not appear substantial. In any case, we will now use the data from the "100" treatment as a benchmark to analyze the focal treatments. The question, then, is whether these results change with the presence of a nonequilibrium focal point.

++++ Table 5 about here ++++

\footnotetext{
${ }^{44}$ One-tailed binomial test, significant at $3.1 \%$ for periods $1-50$.

${ }^{45}$ Wilcoxon signed ranks test, comparing periods $41-50$ with periods 1-10.

${ }^{46}$ One-tailed binomial test, not significant for periods $1-10$, but significant at $1.6 \%$ for periods 41-50 as well as periods 1-50.

47 One-tailed Wilcoxon signed ranks test, comparing periods 41-50 with periods 1-10, significant at $3.1 \%$.

${ }^{48}$ One-tailed binomial test.
} 
Figures 9.a to 9.c present the average number of choices of the focal, associated, and most chosen other actions in the focal treatments. The individual sessions are shown in Table 5. We first look at the average number of choices of the most chosen other action. These averages decrease from period 1-10 to period 41-50 from 3.9 to 3.6 , from 3.5 to 2.8 , and from 3.2 to 1.9 for the "46", "87" and "99" treatments respectively. Thus, they remain at average values well below what would be expected from random behavior (i.e., 4.3).

Experimental observation 18 (50 periods): In none of the focal treatments is the frequency of the most chosen other action higher than what would be expected from random behavior. ${ }^{49}$

Experimental observation 19 (50 periods): In none of the focal treatments does the frequency of the most chosen other action increase over time..$^{50}$

The frequency with which the focal action is chosen increases from periods $1-10$ to periods $41-50$ from 4.0 to 4.6 , from 6.0 to 8.2 , and from 7.8 to 11.5 in the "46", "87" and "99" treatments respectively. Similarly, these frequencies for the associated actions increase from 1.3 to 3.5 , from 1.6 to 4.6 , and from 1.3 to 2.7 . As a result, the number of subjects making use of the focal point (choosing either the focal or associated action) equals eventually 45 , 71 and $79 \%$ in periods $41-50$ in the "46", "87" and "99" treatments respectively.

Experimental observation 20 (50 periods): In the "87" and "99" treatments the frequency that subjects make use of the FP (choosing either the focal or associated action) is higher than what would be expected from random behavior. ${ }^{51}$ In the "46" treatment, this is true as well for periods $1-10$, but not for periods $41-50$, or $1-50 .{ }^{52}$

Experimental observation 21 (50 periods): In all focal treatments the frequency that subjects make use of the FP (choosing either the focal or associated action) increases over time. ${ }^{53}$

We now make a comparison of the actions chosen across the various focal treatments.

\footnotetext{
${ }^{49}$ One-tailed binomial test, considering periods 1-10, 41-50 and 1-50 for the "46", "87" and "99" treatments.

50 One-tailed Wilcoxon signed ranks test, comparing periods 411-50 with periods 1-10. In fact, in the "99" treatment the frequency over the most chosen other action decreases (significant at $1.6 \%$ ).

${ }^{51}$ One-tailed Binomial test, significant at $1.6 \%$ for periods $1-10,41-50$ as well as $1-50$.

${ }^{52}$ One-tailed Binomial test, significant at $3.5 \%$ for periods $1-10$.

53 One-tailed Wilcoxon signed ranks test, comparing periods 41-50 with periods 1-10, significant at 5.5, 1.6 and $3.1 \%$ for the "46", "87" and "99" treatments respectively.
} 
Experimental observation 22 (50 periods): The higher the focal payoff, the more often the focal action itself is chosen, ${ }^{54}$ while there is no such significant difference between the various focal treatments as far as the frequency of the associated actions is concerned. ${ }^{55}$

Experimental observation 23 (50 periods): In the "87" and in the "99" treatments, subjects make use of the FP (choosing either the focal or associated action) significantly more than in the "46" treatment, ${ }^{56}$ while there is no significant difference between the "99" and "87" treatments.

As a measure of the coordination success we look again at the average expected payoffs, allocating them, as above, to the FP, the first and second ANE, and any other NE. These payoffs are represented in Figures 10.a to 10.c. For details on the individual sessions in the "46", "87", and "99" treatments see Table 6.

++++ Table 6 about here ++++

Recall that if the players choose randomly among the NE, the expected payoff is about 13 , and that in the "100" treatment the average expected payoffs in periods $41-50$ stay below 20 (18.1). In periods 41-50, in the "46" treatment the average expected payoff reaches 32.4 , i.e., almost doubling the expected payoffs without focal point in the "100" treatment, in the "87" treatment the average expected payoff reaches 55.2, and in the "99" treatment it reaches a level of 63.5 .

In the "46" treatment, the expected payoffs obtained through the other NE and through the FP stay relatively constant. But this is different for the payoffs obtained through the ANE. In particular, building on the platform provided by the FP, the success of the first ANE increases dramatically. Eventually, in periods 41-50, in the "46" treatment it is one of the ANE that accounts for $70 \%$ of the payoffs related to the FP, while the other ANE is largely ignored (14\%), and only $16 \%$ of the payoffs related to the FP come through the FP itself. A similar pattern can be observed in the "87" treatment. The first ANE is built on the platform of a focal point coordination. Eventually the first ANE leads to $57 \%$ of the expected payoffs related to the FP, while the second ANE provides only $7 \%$ of them, and the FP itself gives $36 \%$ of the expected payoffs related to the FP. Finally, in the "99" treatment, it is the FP that leads early on to considerable payoffs, in the end accounting for $63 \%$ of the expected payoffs related to the FP. One ANE becomes more and more important, providing eventually $33 \%$ of the expected payoffs related to the FP, with the other ANE providing only $4 \%$.

\footnotetext{
${ }^{54}$ One-tailed robust rank-order test for periods $1-50$, significant at $5 \%$ for "87" against "46", $1 \%$ for "99" against "46", and $2.5 \%$ for "99" against "87".

${ }^{55}$ In fact, in periods 41-50, the associated action is chosen significantly less in the "99" than in the "87" treatment (one-tailed robust rank-order at $5 \%$ ).

${ }^{56}$ One-tailed robust rank-order test, significant at 10 and $5 \%$ respectively for periods $1-50$.
} 
Experimental observation 24 (50 periods): The expected payoffs in each of the focal treatments are higher than in the non-focal "100" treatment. ${ }^{57}$

Experimental observation 25 (50 periods): The expected payoffs in each of the focal treatments increase over time. ${ }^{58}$

As the following observations make clear, these higher payoffs are not due to an increase in the expected payoffs achieved through any of the other NE. As Table 6 shows, the average expected payoffs per period generated by the other NE over the 50 periods are 9.4 (evolving from 9.0 in periods $1-10$ to 10.2 in periods $1-50$ ), 5.9 (from 6.3 to 6.7 ) and 2.4 (from 4.2 to 1.5) for the "46", "87" and "99" treatments respectively, whereas they are 15.7 in the "100" treatment.

Experimental observation 26 ( 50 periods): The expected payoffs realized through any of the other NE are lower in the focal treatments than in the non-focal "100" treatment. ${ }^{59}$

Experimental observation 27 ( 50 periods): The expected payoffs realized through any of the other NE do not increase over time in the focal treatments. ${ }^{60}$

In other words, the increased coordination success must be due to coordinating on either the FP or the ANE. This is confirmed by Table 5. Comparing periods 1-10 with periods 41-50, we see that the expected payoffs realized through the FP increase from 2.9 to 3.6 in the "46" treatment, from 9.9 to 17.3 in the "87" treatment, and from 18.3 to 38.8 in the "99" treatment. Similarly, the payoffs generated by the ANE increase from 4.6 to 18.8 , from 5.7 to 28.7 , and from 6.9 to 21.4 in the "46", "87" and "99" treatments respectively. This leads to an increase in the share in the expected payoffs realized through the ANE alone from 28 to $58 \%$ in the "46" treatment, from 28 to $57 \%$ in the "87" treatment, and from 26 to $37 \%$ in the "99" treatment from periods 1-10 to periods 41-50. As a result, the share in the average expected payoffs obtained through either the FP or the ANE reaches $69 \%, 88 \%$ and $98 \%$ for "46", " 87 " and "99" treatments respectively in periods 41-50.

${ }^{57}$ One-tailed robust rank-order test, significant at 10, 1 and less than $1 \%$ for the "46", "87" and "99" treatments respectively for periods 1-50.

58 One-tailed Wilcoxon signed ranks test, significant at $0.4,1.6$, and $1.6 \%$ for the "46", "87" and "99" treatments respectively for periods 41-50 against periods 1-10.

${ }_{59}$ One-tailed robust rank-order test, significant at $2.5 \%$ for the "46" treatment and less than $1 \%$ for the "87" and "99" treatments for periods $1-50$. Similar observations can be made if we average over all the (other) NE.

${ }^{60}$ One-tailed Wilcoxon signed ranks test, comparing periods 41-50 with periods 1-10. In fact, in the "99" treatment the expected payoffs generated by the other NE decrease over time (significant at 6.3\%). 
Experimental observation 28 (50 periods): In the "87" and "99" treatments, the expected payoffs realized through the FP are higher than those coming through the best of the other $\mathrm{NE},{ }^{61}$ while there is no such significant difference in the "46" treatment.

Experimental observation 29 (50 periods): In the "87" and "99" treatments, the expected payoffs realized through the best of the ANE are higher than those coming through the best of the other $\mathrm{NE},{ }^{62}$ while there is no such significant difference in the " 46 " treatment.

Experimental observation 30 (50 periods): In the "87" and "99" treatments, the expected payoffs realized through either the FP or the ANE are higher than those coming through the other NE combined ${ }^{63}$ while there is no such significant difference in the " 46 " treatment.

Experimental observation 31 (50 periods): In the "87" and "99" treatments, the expected payoffs realized through the FP increase over time ${ }^{64}$ while there is no significant difference in the "46" treatment.

Experimental observation 32 (50 periods): In all focal treatments, the expected payoffs realized through the ANE increase over time. ${ }^{65}$

Experimental observation 33 (50 periods): In all focal treatments, the expected payoffs realized through either the FP or the ANE increase over time. ${ }^{66}$

Experimental observation 34 (50 periods): In the "46" and "87" treatments, the share of the expected payoffs realized through the ANE increases over time,${ }^{67}$ while there is no significant difference in the "99" treatment.

The next two observations concern exclusively the expected payoffs realized by any of the ANE. The question is to what extent one of the two ANE becomes dominant. Therefore, we

\footnotetext{
${ }^{61}$ Wilcoxon signed ranks test, significant at $1.6 \%$ for periods $1-50$.

${ }^{62}$ Wilcoxon signed ranks test, significant at 3.1 and $1.6 \%$ respectively for periods $1-50$.

${ }^{63}$ Wilcoxon signed ranks test, significant at 3.1 and $1.6 \%$ respectively for periods $1-50$.

${ }^{64}$ Wilcoxon signed ranks test, significant at 3.1 and $4.7 \%$ respectively for periods $1-50$ against periods 1-10.

${ }^{65}$ Wilcoxon signed ranks test, significant at 5.5, 1.6 and $1.6 \%$ for the "46", "87" and "99" treatments respectively for periods $41-50$ against periods $1-10$.

${ }^{66}$ Wilcoxon signed ranks test, significant at 5.5, 1.6 and 1.6\% for the "46", "87" and "99" treatments respectively for periods 1-50 against periods 1-10.

${ }^{67}$ Wilcoxon signed ranks test, significant at 9.8 and $1.6 \%$ respectively for periods $41-50$ against periods $1-10$.
} 
consider the relative share of the expected payoffs realized by each of the ANE (see Figure 11 and Table 7). ${ }^{68}$

Experimental observation 35 (50 periods): The one-sided 95\% confidence interval for the median value of the relative share of the expected payoffs realized through the first ANE in periods $41-50$ ranges from 1.00 down to 0.48 , from 1.00 down to 0.55 , and from 1.00 down to 0.43 in the "46", "87" and "99" treatments respectively. ${ }^{69}$

Experimental observation 36 (50 periods): The relative share of the expected payoffs realized through the first ANE increases over time in the "87" treatment but not in the "46" or "99" treatments. ${ }^{70}$

We now turn to making a comparison between the various focal treatments.

Experimental observation 37 (50 periods): The expected payoffs in the "87" and "99" treatments are higher than in the " 46 " treatment, ${ }^{71}$ while there is no significant difference between the "87" and "99" treatments.

The next observation shows that the larger the difference between the payoff of the NE and the payoff of the non-equilibrium focal point, the more frequent is the use of the focal point as an equilibrium selection device rather than as the action of choice.

Experimental observation 38 (50 periods): The higher the focal payoff, the higher the share of the expected payoffs realized through the FP relative to the ANE. ${ }^{72}$

\footnotetext{
68 That is, the expected payoffs realized through the first ANE divided by the expected payoffs realized through either the first or second ANE.

${ }^{69}$ One-tailed binomial test.

${ }^{70}$ Wilcoxon signed ranks test, comparing periods $41-50$ with periods $1-10$, significant at $7.8 \%$ in the "87" treatment.

${ }^{71}$ One-tailed robust rank-order test, significant at 5 and $1 \%$ respectively for periods $1-50$.

72 One-tailed robust rank-order test, significant in periods $1-50$ at 1 , less than 1 and $1 \%$ for "87" against "46", "99" against "46" and "99" against "87" respectively.
} 


\section{Appendix 2: Instructions and screenshots}

We first present the English version of the instructions. In the experiments we actually used a Spanish version (available from the authors upon request).

\section{Introduction}

- We will do a series of 2 experiments. The specific instructions for the second experiment will follow later, once the first experiment is over. The instructions for these experiments are simple, and if you pay attention, you can gain some money that will be paid to you at the end of the series of experiments. From now on till the end of this experimental session you are not allowed to communicate with each other. If you have a question, please raise your hand.

\section{Experiment I}

- There will be two equally sized groups of players ("yellow" and "blue"). Allocation to these groups will take place randomly and anonymously. Therefore, apart from knowing your own color, you will never be told who was in which group.

- The first experiment involves playing a game with another participant. To make pairs of players, each "yellow" player will be randomly and anonymously matched to a "blue" player. Note that you will never know the identity of the person you are matched with, nor will (s)he be aware of yours. Nor will you be told the payoffs of any other person.

- You will get a payoff table showing the various payoff levels that you and the other player can each obtain depending upon the action chosen by you and the other player. All players in this experiment will face the same payoff table.

- The actions for the "blue" players are shown on the rows, and for the "yellow" players on the columns.

- In each round, all players simultaneously choose an action by clicking on the row (for "blue" players) or column ("yellow" players) button of their choice.

- The resulting payoffs for both players are shown within the body of the table by the intersection of the actions chosen. The numbers in the table represent the payoffs in points.

- The points earned in this experiment I will be exchanged into Euros at the end of the session using the following exchange rate: Euro 1.00 per 10 points.

- There is no time limit for your decisions.

- We will do, next, a simple exercise with a fake payoff table to verify that you understand how the payoff table works. 


\section{Exercise with fake payoff table}

Look at the payoff table below, and complete the following sentence:

If the white player chooses row $\mathrm{X}$ and the grey player chooses column $\mathrm{Y}$, then the payoff to the white player is: and the payoff to the grey player is:

\begin{tabular}{||c||c|c|c|c|}
\hline & $Y$ & & & \\
\hline \hline & 1 & 2 & 3 & 4 \\
\hline \hline & 5 & 6 & 7 & 8 \\
\hline \hline$X$ & 9 & 10 & 11 & 12 \\
\hline \hline & 13 & 14 & 15 & 16 \\
\hline
\end{tabular}




\section{Experiment II}

- We will continue with the same "yellow" and "blue" groups as in experiment I. The colors assigned will remain the same throughout the experiment.

- In this second experiment, you will play 50 rounds of the same basic two-player game as in experiment I. This time you will be randomly and anonymously matched to a player in the other group in each round anew.

- As in the previous experiment, you will get a payoff table showing the various payoff levels that you and the other player can each obtain depending upon the action chosen by you and the other player. All players in this experiment will face the same payoff table.

- As in the previous experiment, the actions for the "blue" players are shown on the rows, while the actions for the "yellow" players are shown on the columns.

- As in the previous experiment, in each round, all players simultaneously choose an action by clicking on the row (for "blue" players) or column ("yellow" players) button of their choice.

- As in the previous experiment, the resulting payoffs for both players are shown within the body of the table by the intersection of the actions chosen. The numbers in the table represent payoffs in points.

- The points earned in experiment II will be exchanged into Euros at the end of the session using the following exchange rate: Euro 0.05 per 10 points. Note that the exchange rate is different in this experiment.

- $\quad$ There is no time limit for your decisions.

- After each round, you will be told the points you earned in that round, as well as the total amount of points earned in experiment II up to that round.

Next, we show two sample screenshots from one session of the one-shot experiments. Figure 12.a shows the screen of a (blue) row player, while Figure 12.b shows the screen of the corresponding (yellow) column player.

++++ Figure 12 about here ++++ 


\section{References}

Bacharach, Michael (1993). Variable Universe Games. In Ken Binmore et al. (eds.) Frontiers of Game Theory. Cambridge, MA, MIT Press.

Bacharach, Michael (2006). Beyond Individual Choice, Teams and Frames in Game Theory, Natalie Gold and Robert Sugden, eds., Princeton University Press.

Bacharach, Michael and Michele Bernasconi (1997). The Variable Frame Theory of Focal Points: An Experimental Study. Games and Economic Behavior 19, 1-45.

Bardsley, Nicholas, Judith Mehta, Chris Starmer and Robert Sugden (2006). The Nature of Salience Revisited: Cognitive Hierarchy Theory versus Team Reasoning. WP, U. of Nottingham.

Binmore, Ken, Joe Swierzbinski, Steven Hsu and Chris Proulx (1993). Focal Points and Bargaining, international Journal of Game Theory 22, 4, 381-409.

Binmore, Ken and Larry Samuelson (2006) The Evolution of Focal Points", Games and Economic Behavior 55, 21-42

Blume, Andreas and Uri Gneezy (2000). An Experimental Investigation on Optimal Learning in Coordination Games. Journal of Economic Theory 90, 161-172.

Bosch-Domènech, Antoni, José García-Montalvo, Rosemarie Nagel, Albert Satorra (2002) "One, Two, (Three), Infinity...: A Comparison of Behavior of Newspaper Readers, Game Theorists, and Students in Beauty-Contest Experiments", American Economic Review, 92-5, 1688-1701.

Camerer, Colin F., Teck-Hua Ho and Juin-Kuan Chong (2004). A Cognitive Hierarchy Model of Games. Quarterly Journal of Economics 119 (3), 861-98.

Casajus, André (2001). Focal Points in Framed Games - Breaking the Symmetry (Lecture Notes in Economics and Mathematical Systems 499). Berlin: Springer.

Crawford, Vincent P. and Hans Haller (1990). Learning How to Cooperate: Optimal Play in Repeated Coordination Games. Econometrica 58, 571-595.

Crawford, Vincent P. and Nagore Iriberri (2007a). Level-k Auctions: Can a Non-Equilibrium Model of Strategic Thinking Explain the Winner's Curse and Overbidding in Private Value Auctions? Econometrica 75, 6, 1721-70.

Crawford, Vincent P. and Nagore Iriberri (2007b). Salience, Naiveté, and Sophistication in Experimental "Hide-and-Seek" Games. American Economic Review 97 (5), 1731-1750.

Crawford, Vincent, Uri Gneezy and Yuval Rottenstreich (2007). The Power of Focal Points is Limited: Even Minor Payoff Asymmetry Yields Massive Coordination Failures, Preliminary draft.

Cooper, Russel W., Douglas V. DeJong, Robert Forsythe and Thomas W. Ross (1990). Selection Criteria in Coordination Games: Some Experimental Results. American Economic Review 80, 218-33.

Devetag, M. Giovanna (2000). Cognition, Interactions and Macrobehaviors: Four Essays (Ph.D. thesis) Scuola Superiore Sant'Anna, Pisa.

Gauthier, D. (1975). Coordination. Dialogue 14, 195-221.

Gilbert, Margaret (1989). Rationality and Salience. Philosophical Studies 57, 61-77.

Harrison, Glenn W. (2005). Field Experiments and Control, in T. Carpenter, G. W. Harrison and J. A. List (eds.), Field Experiments in Economics, Greenwich, CT, JAI Press, Research in Experimental Economics, Vol. 10, 17-50.

Harsanyi, John and Reinhard Selten, 1988, A General Theory of Equilibrium Selection in Games, Cambridge: MIT Press 
Huber Joel, John Payne and Christopher Puto, 1982, Adding Asymmetrically Dominated Alternatives. Violations of Regularity and the Similarity Hypothesis, Journal of Consumer Research 9, 90-98.

Janssen, Maarten (2001). Rationalizing Focal Points. Theory and Decision 50, 119-148.

Ledyard, John O. (1995). Public Goods: A Survey of Experimental Research. In J.H. Kagel \& A.E. Roth (Eds.), Handbook of Experimental Economics (pp. 111-194). Princeton, NJ: Princeton University Press.

McAdams, Richard H. (2000). A Focal Point Theory of Expressive Law, Virginia Law Review 86, 8, 1649-1729.

Mehta, Judith, Chris Starmer and Robert Sugden (1994). The Nature of Salience: An Experimental Investigation of Pure Coordination Games. American Economic Review 84 (3), $658-73$.

Nagel, Rosemarie (1995). Unraveling in Guessing Games: An Experimental Study, American Economic Review 85, 5, 1313-26.

Offerman, Theo (1997). Beliefs and Decision Rules in Public Good Games. Theory and Experiments. Dordrecht: Kluwer.

Schelling, Thomas C. (1960). The Strategy of Conflict. Cambridge, MA, Harvard University Press.

Stahl, Dale O. II and Paul W. Wilson (1995). On Players' Models of Other Players: Theory and Experimental Evidence, Games and Economic Behavior 10(1), 218-54.

Sugden, Robert (1995). A Theory of Focal Points. Economic Journal, 105, 533-550.

Sugden, Robert and Ignacio Zamarrón (2006). Finding the Key: The Riddle of Focal Points. Journal of Economic Psychology 27, 609-621.

Young, H. Peyton (1993), The Evolution of Conventions, Econometrica 61, 1, 57-84. 
Figure 1. Coordination game, Description 0

\begin{tabular}{|c|c|c|c|c|c|c|c|c|c|c|c|c|c|c|}
\hline 0 & 0 & 0 & 0 & 0 & 0 & 0 & 0 & 100 & 0 & 0 & 100 & 0 & 0 & 0 \\
\hline 0 & 0 & 0 & 0 & 0 & 100 & 0 & 0 & 0 & 0 & 0 & 0 & 0 & 0 & 100 \\
\hline 0 & 0 & 0 & 100 & 0 & 0 & 0 & 100 & 0 & 0 & 0 & 0 & 0 & 0 & 0 \\
\hline 0 & 0 & 0 & 0 & 100 & 0 & 100 & 0 & 0 & 0 & 0 & 0 & 0 & 0 & 0 \\
\hline 0 & 0 & 0 & 0 & 0 & 0 & 0 & 100 & 0 & 0 & 0 & 0 & 100 & 0 & 0 \\
\hline 0 & 100 & 0 & 0 & 0 & 0 & 0 & 0 & 0 & 0 & 0 & 0 & 0 & 100 & 0 \\
\hline 0 & 0 & 100 & 0 & 0 & 0 & 0 & 0 & 0 & 0 & 0 & 0 & 100 & 0 & 0 \\
\hline 100 & 0 & 0 & 0 & 0 & 0 & 0 & 0 & 0 & 0 & 0 & 0 & 0 & 0 & 100 \\
\hline 0 & 100 & 0 & 0 & 0 & 0 & 0 & 0 & 0 & 0 & 100 & 0 & 0 & 0 & 0 \\
\hline 0 & 0 & 0 & 0 & 0 & 0 & 0 & 0 & 0 & 0 & 0 & 100 & 0 & 100 & 0 \\
\hline 0 & 0 & 100 & 0 & 0 & 0 & 0 & 0 & 0 & 100 & 0 & 0 & 0 & 0 & 0 \\
\hline 0 & 0 & 0 & 0 & 100 & 0 & 0 & 0 & 100 & 0 & 0 & 0 & 0 & 0 & 0 \\
\hline 100 & 0 & 0 & 0 & 0 & 0 & 0 & 0 & 0 & 0 & 100 & 0 & 0 & 0 & 0 \\
\hline 0 & 0 & 0 & 0 & 0 & 100 & 0 & 0 & 0 & 100 & 0 & 0 & 0 & 0 & 0 \\
\hline 0 & 0 & 0 & 100 & 0 & 0 & 100 & 0 & 0 & 0 & 0 & 0 & 0 & 0 & 0 \\
\hline
\end{tabular}


Figure 2. Coordination game, Description 0

\begin{tabular}{|c|c|c|c|c|c|}
\hline & $\begin{array}{c}\text { first } \\
\text { white } \\
\text { ball }\end{array}$ & $\begin{array}{c}\text { second } \\
\text { white } \\
\text { ball }\end{array}$ & $\begin{array}{c}\text { third } \\
\text { white } \\
\text { ball }\end{array}$ & $\begin{array}{c}\text { fourth } \\
\text { white } \\
\text { ball }\end{array}$ & red ball \\
\hline $\begin{array}{c}\text { first white } \\
\text { ball }\end{array}$ & 100 & 0 & 0 & 0 & 0 \\
\hline $\begin{array}{c}\text { second } \\
\text { white ball }\end{array}$ & 0 & 100 & 0 & 0 & 0 \\
\hline $\begin{array}{c}\text { third white } \\
\text { ball }\end{array}$ & 0 & 0 & 100 & 0 & 0 \\
\hline $\begin{array}{c}\text { fourth } \\
\text { white ball }\end{array}$ & 0 & 0 & 0 & 100 & 0 \\
\hline red ball & 0 & 0 & 0 & 0 & 80 \\
\hline
\end{tabular}

Figure 3. Transformed game, Description 1

\begin{tabular}{|c|c|c|c|}
\hline & $\begin{array}{c}\text { white } \\
\text { ball }\end{array}$ & $\begin{array}{c}\text { red } \\
\text { ball }\end{array}$ & $\begin{array}{c}\text { any } \\
\text { ball }\end{array}$ \\
\hline $\begin{array}{c}\text { white } \\
\text { ball }\end{array}$ & 25 & 0 & 20 \\
\hline $\begin{array}{c}\text { red } \\
\text { ball }\end{array}$ & 0 & 80 & 16 \\
\hline $\begin{array}{c}\text { any } \\
\text { ball }\end{array}$ & 20 & 16 & 19.2 \\
\hline
\end{tabular}


Figure 4. Transformed game, Description 1

\begin{tabular}{|c|c|c|c|}
\hline & $\begin{array}{c}\text { column } \\
\text { with focal } \\
\text { point }\end{array}$ & $\begin{array}{c}\text { any } \\
\text { column } \\
\text { with 2 NE }\end{array}$ & $\begin{array}{c}\text { any } \\
\text { column }\end{array}$ \\
\hline $\begin{array}{c}\text { row with } \\
\text { focal point }\end{array}$ & 87 & 7.14 & 12.47 \\
\hline $\begin{array}{c}\text { any row } \\
\text { with 2 NE }\end{array}$ & 7.14 & 13.78 & 13.33 \\
\hline any row & 12.47 & 13.33 & 13.28 \\
\hline
\end{tabular}

Figure 5. Transformed game, Description 2

\begin{tabular}{|c|c|c|c|c|c|}
\hline & $\begin{array}{c}\text { column } \\
\text { with focal } \\
\text { point }\end{array}$ & $\begin{array}{c}\text { associated } \\
\text { column with } \\
\text { 2 NE }\end{array}$ & $\begin{array}{c}\text { other } \\
\text { column } \\
\text { with 2 NE }\end{array}$ & $\begin{array}{c}\text { any column } \\
\text { with 2 NE }\end{array}$ & any column \\
\hline $\begin{array}{c}\text { row with } \\
\text { focal point }\end{array}$ & 87 & 100 & 0 & 7.14 & 12.47 \\
\hline $\begin{array}{c}\text { associated } \\
\text { row with } \\
\text { 2 NE }\end{array}$ & 100 & 0 & 7.69 & 7.14 & 13.33 \\
\hline $\begin{array}{c}\text { other row } \\
\text { with 2 NE }\end{array}$ & 0 & 7.69 & 14.79 & 14.29 & 13.33 \\
\hline $\begin{array}{c}\text { any row with } \\
\text { 2 NE }\end{array}$ & 7.14 & 7.14 & 14.29 & 13.78 & 13.33 \\
\hline any row & 12.47 & 13.33 & 13.33 & 13.33 & 13.28 \\
\hline
\end{tabular}


Figure 6. one-shot: average numbers of choices per session

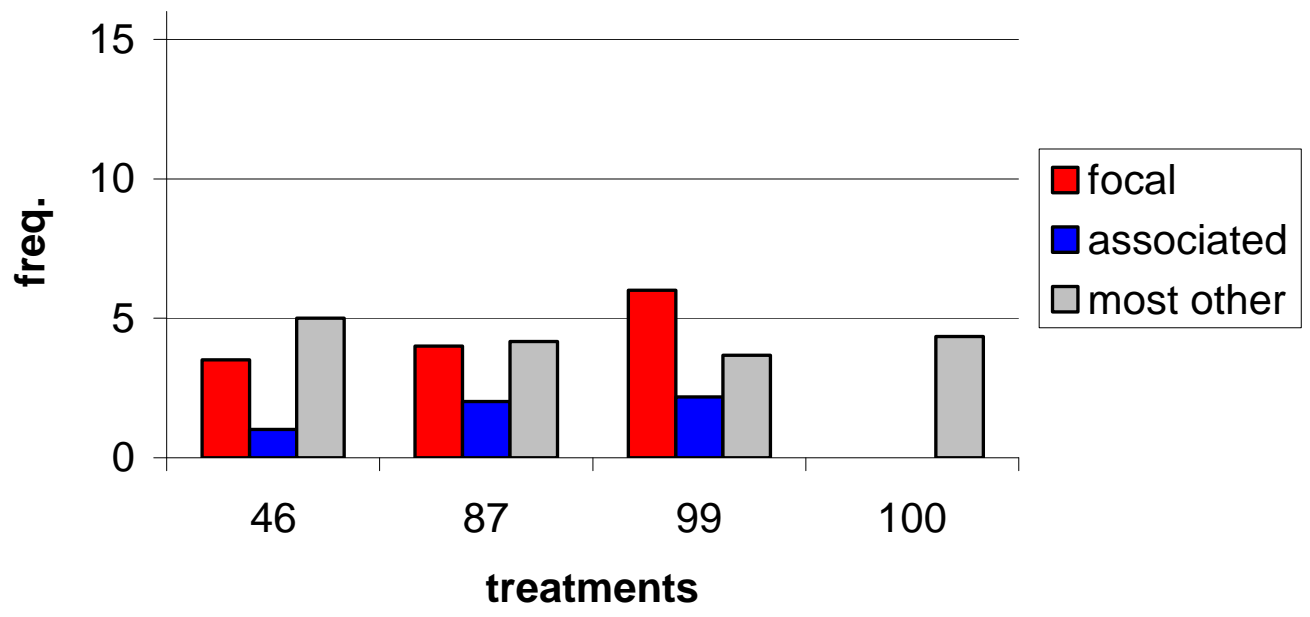


Figure 7. 50 periods: most frequently chosen action in "100" treatment

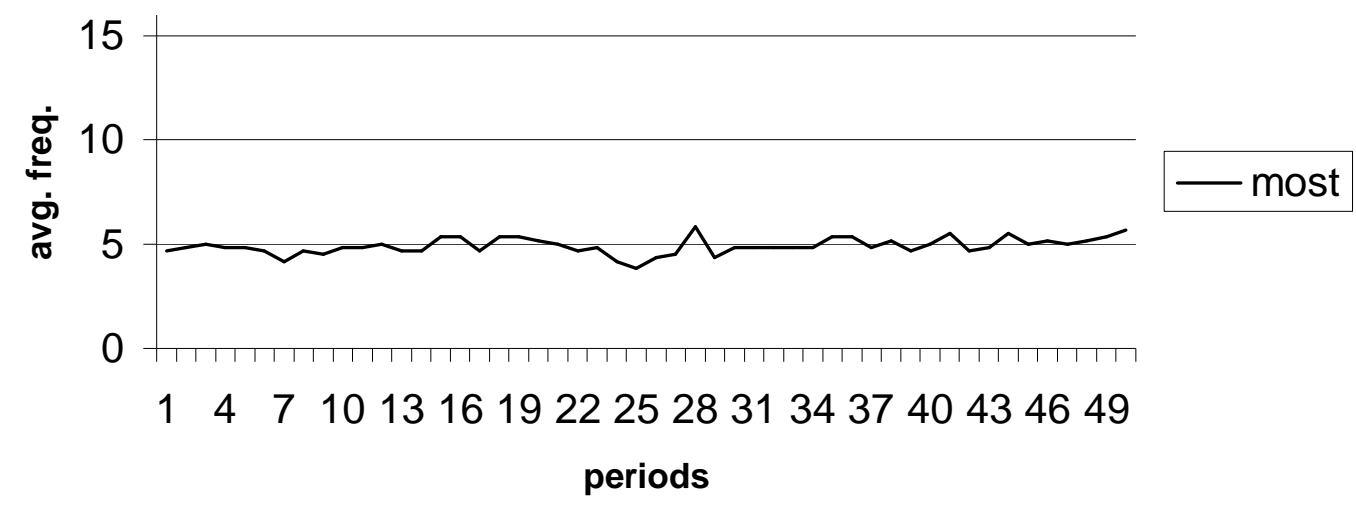


Figure 8. 50 periods: average expected payoffs in "100" treatment

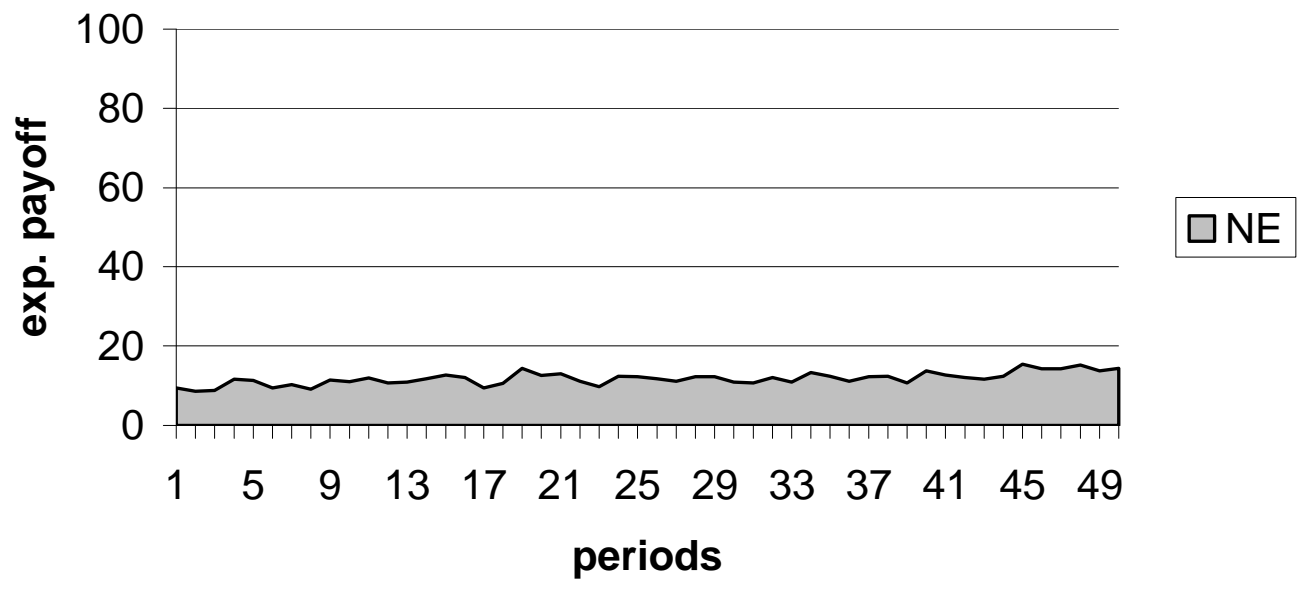


Figure 9.a. 50 periods: actions in "46" treatment

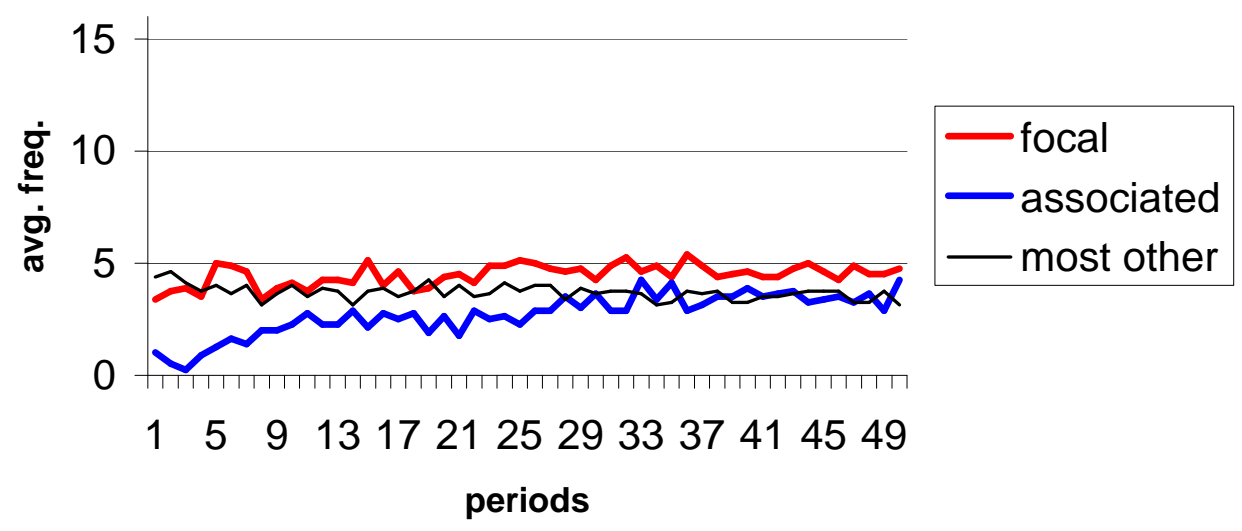

Figure 9.b. 50 periods: actions in " 87 " treatment

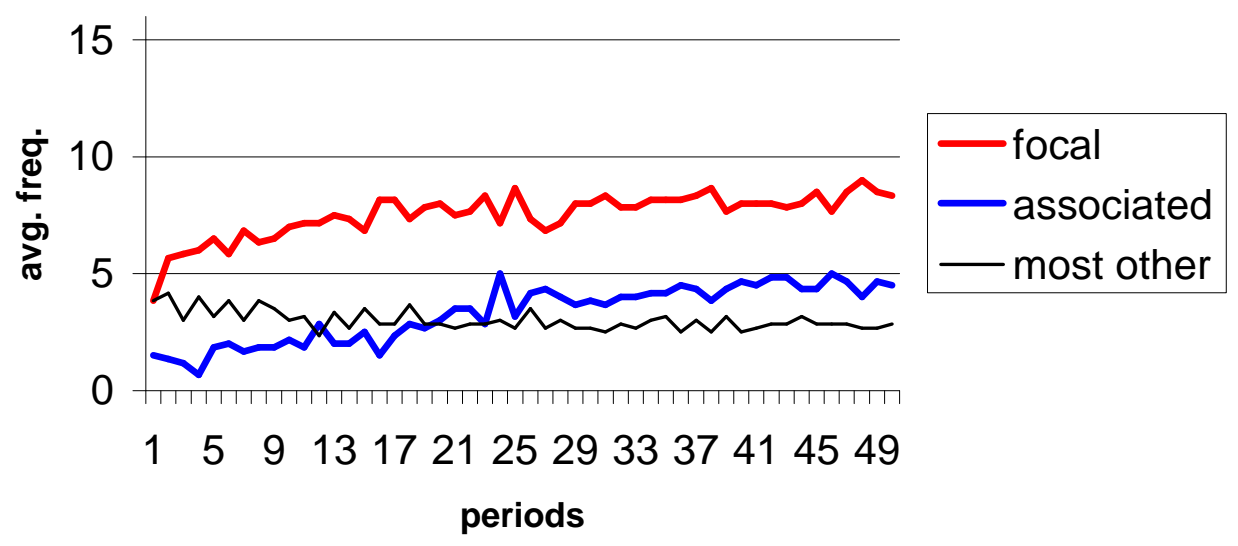

Figure 9.c. 50 periods: actions in "99" treatment

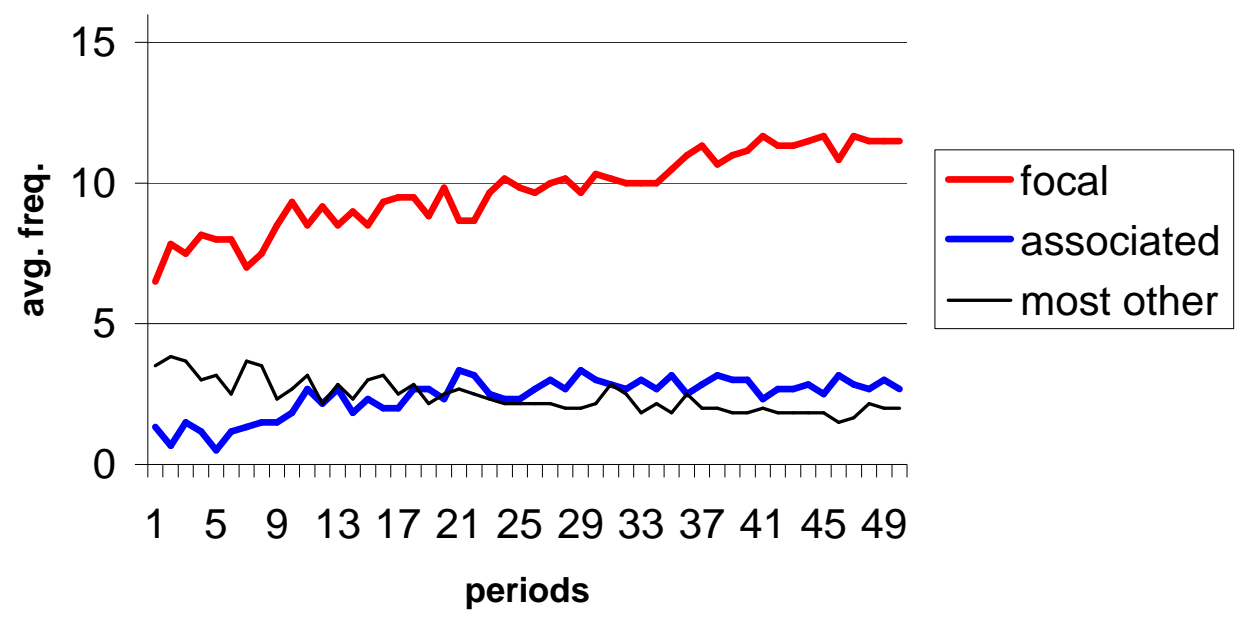


Figure 10.a. 50 periods: average expected payoffs in " 46 " treatment

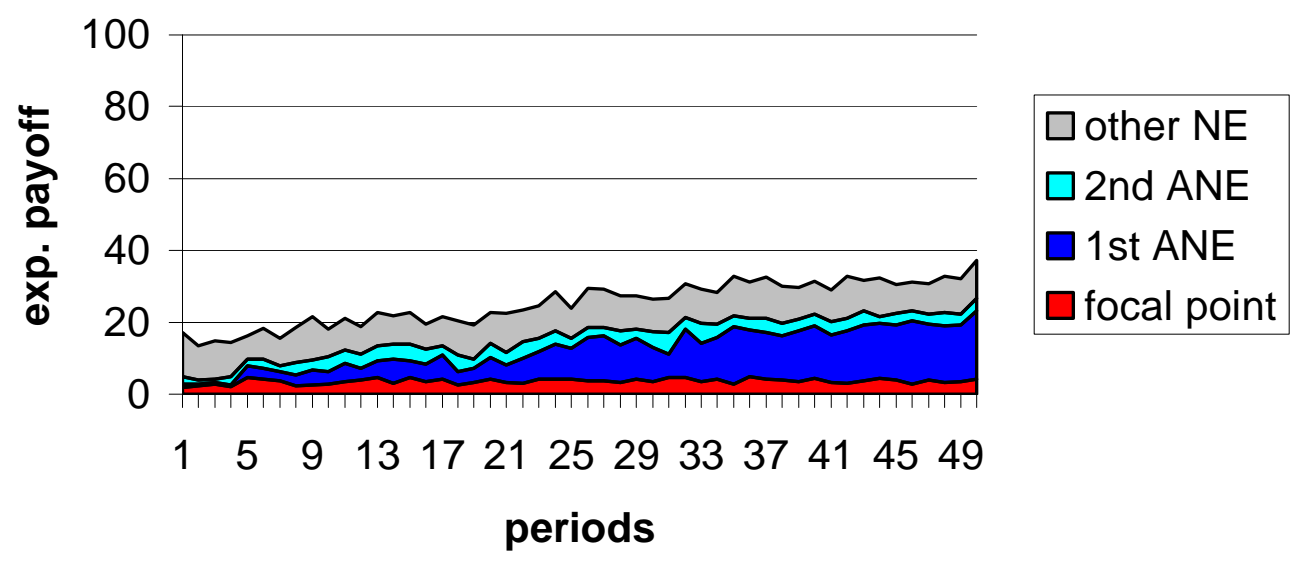

Figure 10.b. 50 periods: average expected payoffs in "87" treatment

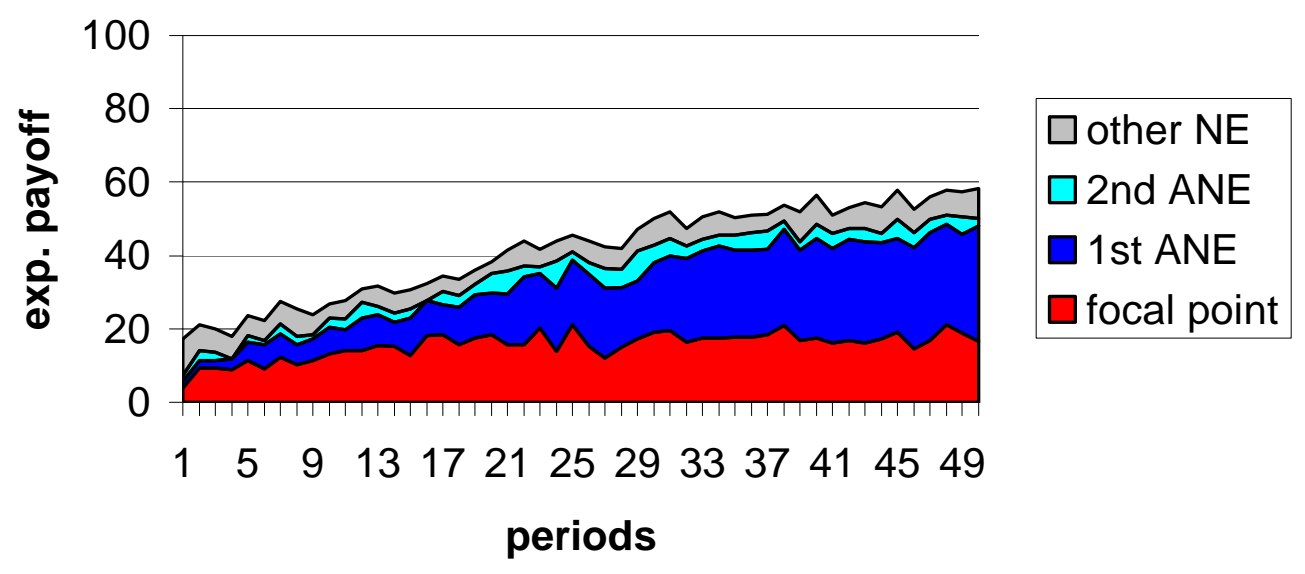

Figure 10.c. 50 periods: average expected payoffs in "99" treatment

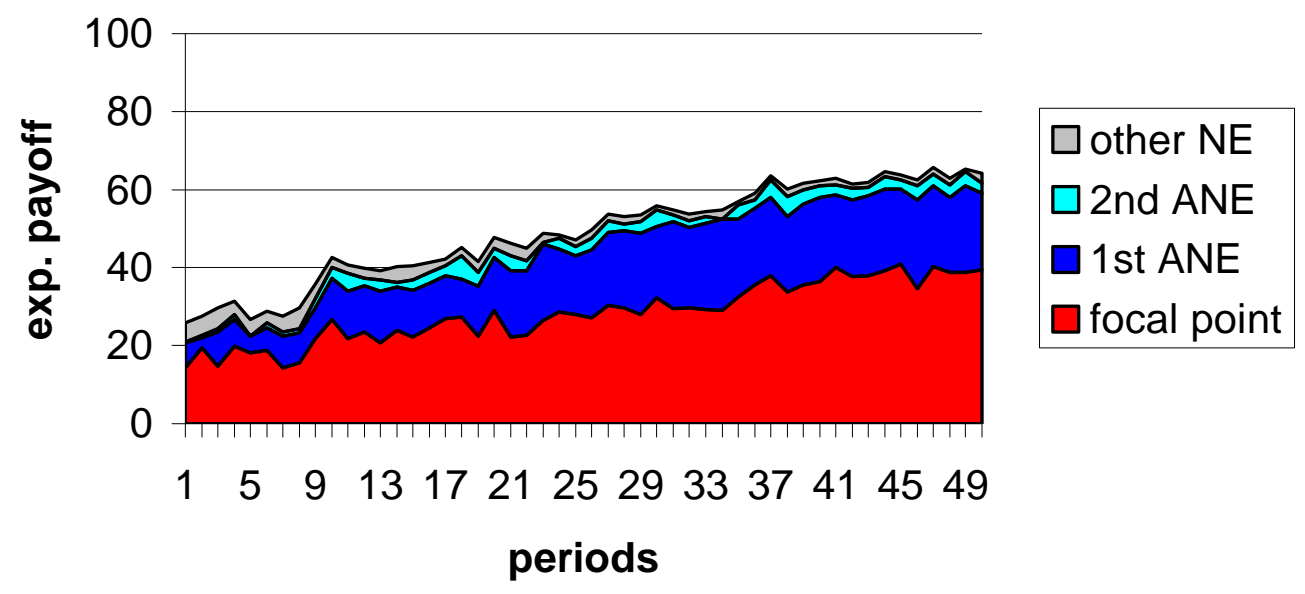


Figure 11.a. 50 periods: relative shares of average expected payoffs for each ANE in "46" treatment

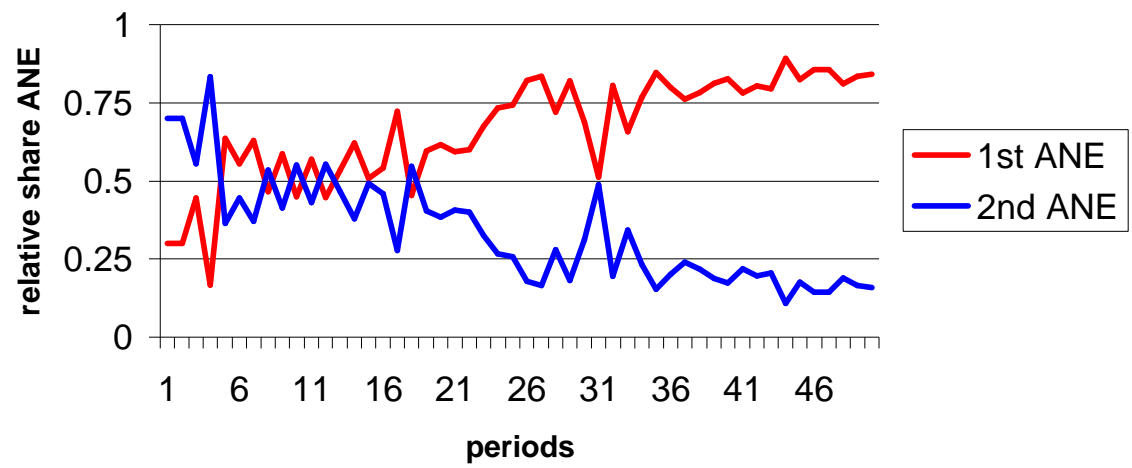

Figure 11.b. 50 periods: relative shares of average expected payoffs for each ANE in "87" treatment

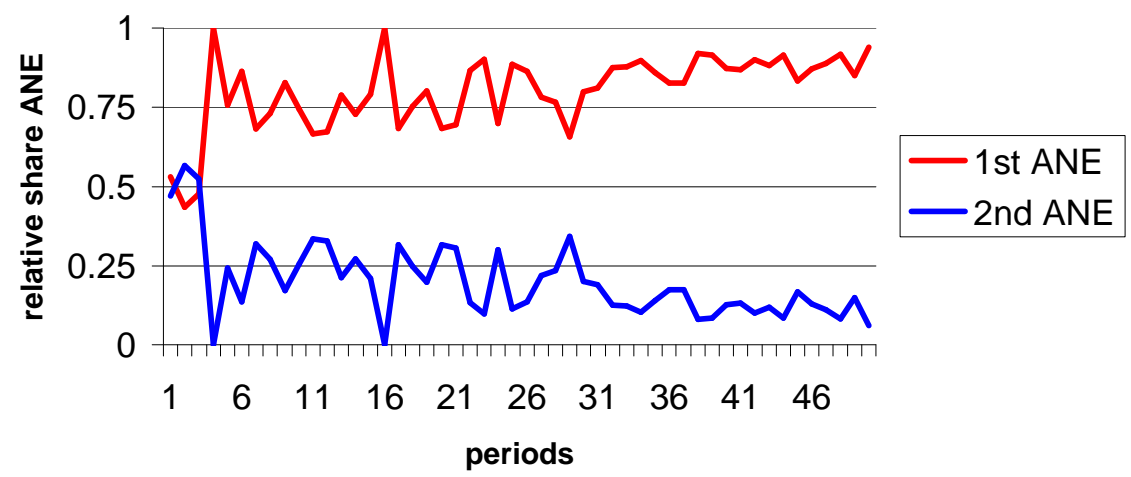

Figure 11.c. 50 periods: relative shares of average expected payoffs for each ANE in "99" treatment

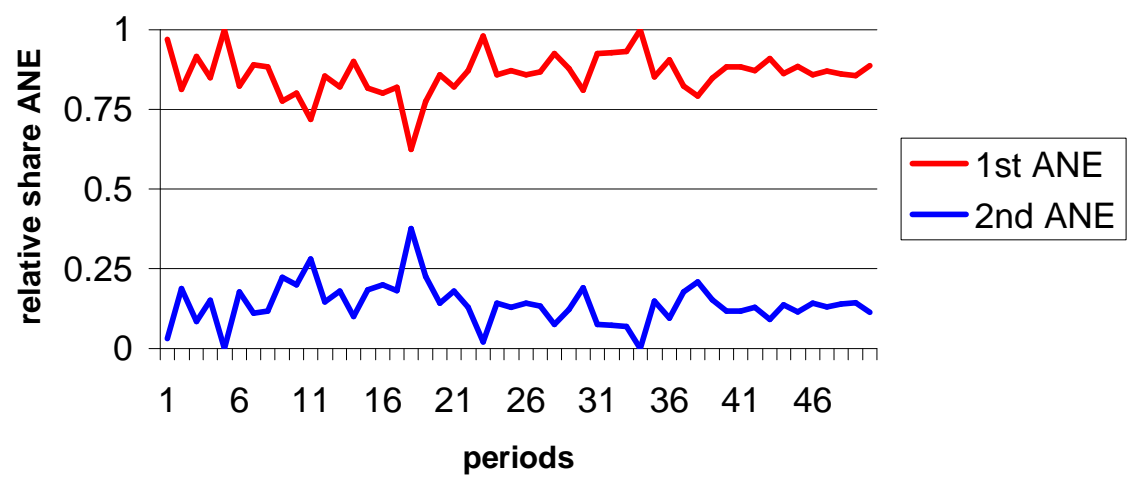


Figure 12.a. Screenshot for row player in one-shot game

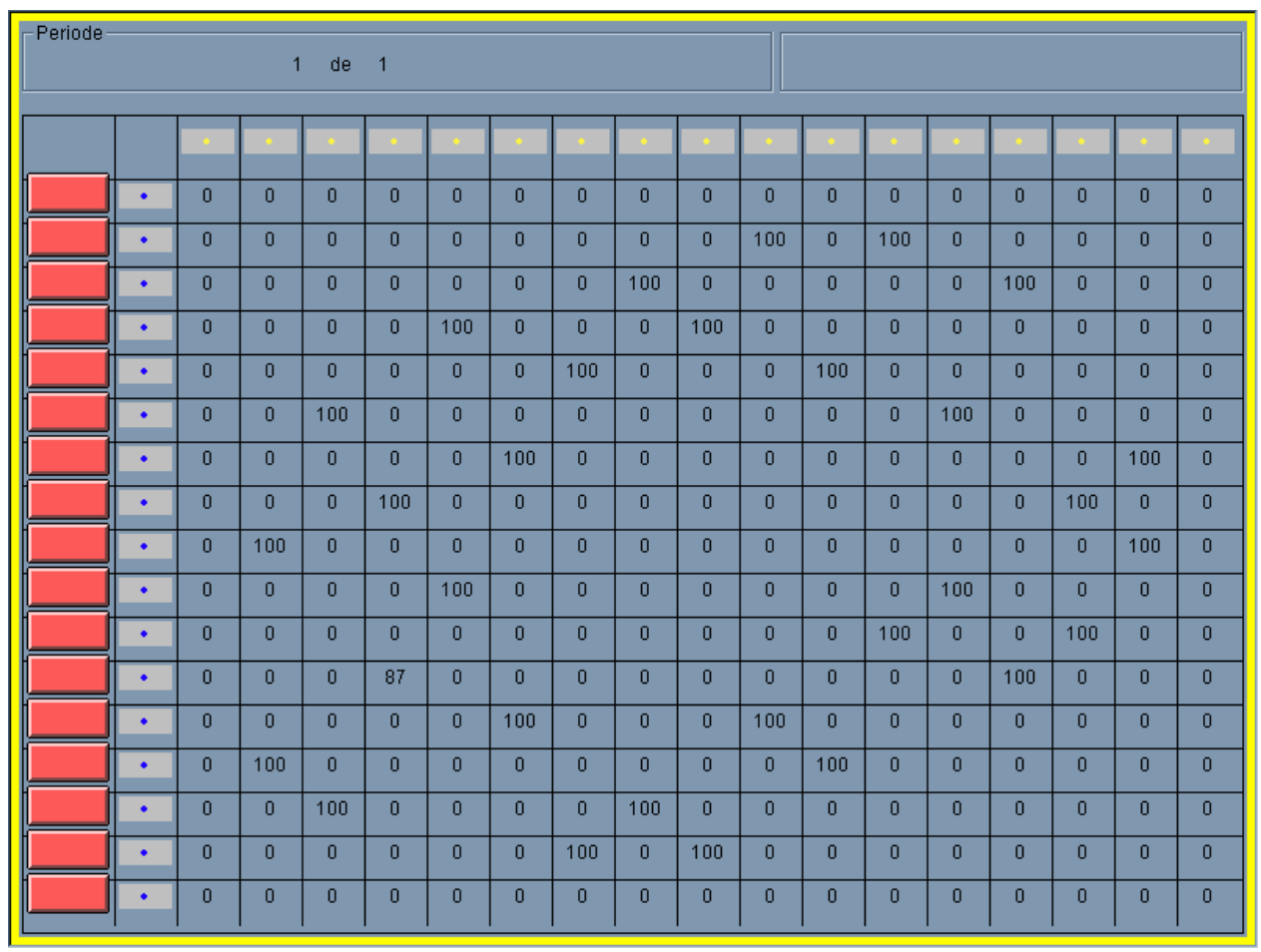

Figure 12.b. Screenshot for column player in one-shot game

\begin{tabular}{|c|c|c|c|c|c|c|c|c|c|c|c|c|c|c|c|c|c|}
\hline \multicolumn{18}{|c|}{ Periode } \\
\hline & & & 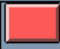 & $\square$ & 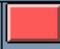 & & E & $\square$ & $\square$ & & $\square$ & $\bar{\square}$ & & E & $\square$ & E & - \\
\hline & . & . & • & • & 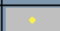 & $\theta$ & $\because$ & - & 7 & ? & 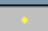 & 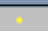 & 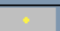 & $\%$ & 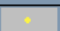 & $\because$ & 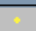 \\
\hline$\cdot$ & 0 & 0 & 0 & 0 & 0 & 0 & 0 & 0 & 0 & 0 & 0 & 0 & 0 & 0 & 0 & 0 & 0 \\
\hline$\cdot$ & 0 & 0 & 0 & 0 & 0 & 0 & 0 & 0 & 0 & 100 & 0 & 100 & 0 & 0 & 0 & 0 & 0 \\
\hline$\cdot$ & 0 & 0 & 0 & 0 & 0 & 0 & 0 & 100 & 0 & 0 & 0 & 0 & 0 & 100 & 0 & 0 & 0 \\
\hline$\cdot$ & 0 & 0 & 0 & 0 & \begin{tabular}{|l|}
100 \\
\end{tabular} & 0 & 0 & 0 & 100 & 0 & 0 & 0 & 0 & 0 & 0 & 0 & 0 \\
\hline$\cdot$ & 0 & 0 & 0 & 0 & 0 & 0 & 100 & 0 & 0 & 0 & 100 & 0 & 0 & 0 & 0 & 0 & 0 \\
\hline$\cdot$ & 0 & 0 & \begin{tabular}{|l|}
100 \\
\end{tabular} & 0 & 0 & 0 & 0 & 0 & 0 & 0 & 0 & 0 & 100 & 0 & 0 & 0 & 0 \\
\hline$\cdot$ & 0 & 0 & 0 & 0 & 0 & 100 & 0 & 0 & 0 & 0 & 0 & 0 & 0 & 0 & 0 & 100 & 0 \\
\hline$\cdot$ & 0 & 0 & 0 & 100 & 0 & 0 & 0 & 0 & 0 & 0 & 0 & 0 & 0 & 0 & 100 & 0 & 0 \\
\hline$\cdot$ & 0 & \begin{tabular}{|l|}
100 \\
\end{tabular} & 0 & 0 & 0 & 0 & 0 & 0 & 0 & 0 & 0 & 0 & 0 & 0 & 0 & 100 & 0 \\
\hline$\cdot$ & 0 & 0 & 0 & 0 & \begin{tabular}{|l|}
100 \\
\end{tabular} & 0 & 0 & 0 & 0 & 0 & 0 & 0 & 100 & 0 & 0 & 0 & 0 \\
\hline$\cdot$ & 0 & 0 & 0 & 0 & 0 & 0 & 0 & 0 & 0 & 0 & 0 & 100 & 0 & 0 & 100 & 0 & 0 \\
\hline$\cdot$ & 0 & 0 & 0 & 87 & 0 & 0 & 0 & 0 & 0 & 0 & 0 & 0 & 0 & 100 & 0 & 0 & 0 \\
\hline$\cdot$ & 0 & 0 & 0 & 0 & 0 & 100 & 0 & 0 & 0 & 100 & 0 & 0 & 0 & 0 & 0 & 0 & 0 \\
\hline$\cdot$ & 0 & \begin{tabular}{|l|l|}
100 \\
\end{tabular} & 0 & 0 & 0 & 0 & 0 & 0 & 0 & 0 & 100 & 0 & 0 & 0 & 0 & 0 & 0 \\
\hline$\cdot$ & 0 & 0 & \begin{tabular}{|l|}
100 \\
\end{tabular} & 0 & 0 & 0 & 0 & 100 & 0 & 0 & 0 & 0 & 0 & 0 & 0 & 0 & 0 \\
\hline$\cdot$ & 0 & 0 & 0 & 0 & 0 & 0 & 100 & 0 & 100 & 0 & 0 & 0 & 0 & 0 & 0 & 0 & 0 \\
\hline$\cdot$ & 0 & 0 & 0 & 0 & 0 & 0 & 0 & 0 & 0 & 0 & 0 & 0 & 0 & 0 & 0 & 0 & 0 \\
\hline
\end{tabular}


Table 1. average earnings per player across treatments

\begin{tabular}{|c|c|}
\hline treatment & average earnings $(\boldsymbol{\epsilon})$ \\
\hline 46 & 8.39 \\
87 & 12.27 \\
99 & 14.82 \\
100 & 5.32 \\
\hline
\end{tabular}


Table 2. one-shot: numbers of choices in individual sessions

\begin{tabular}{|c|c|c|c|}
\hline \multirow[b]{2}{*}{ session } & \multicolumn{3}{|c|}{ frequencies } \\
\hline & focal & associated & most other \\
\hline 46.1 & 1 & 1 & 7 \\
\hline 46.2 & 2 & 1 & 5 \\
\hline 46.3 & 10 & 2 & 3 \\
\hline 46.4 & 4 & 1 & 6 \\
\hline 46.5 & 3 & 1 & 4 \\
\hline 46.6 & 1 & 1 & 4 \\
\hline 46.7 & 3 & 0 & 6 \\
\hline 46.8 & 4 & 1 & 5 \\
\hline avg. & 3.5 & 1.0 & 5.0 \\
\hline 87.1 & 4 & 3 & 4 \\
\hline 87.2 & 2 & 2 & 6 \\
\hline 87.3 & 6 & 0 & 3 \\
\hline 87.4 & 4 & 4 & 4 \\
\hline 87.5 & 5 & 3 & 3 \\
\hline 87.6 & 3 & 0 & 5 \\
\hline avg. & 4.0 & 2.0 & 4.2 \\
\hline 99.1 & 2 & 2 & 4 \\
\hline 99.2 & 6 & 2 & 4 \\
\hline 99.3 & 11 & 5 & 1 \\
\hline 99.4 & 6 & 1 & 3 \\
\hline 99.5 & 8 & 2 & 4 \\
\hline 99.6 & 3 & 1 & 6 \\
\hline avg. & 6.0 & 2.2 & 3.7 \\
\hline 100.1 & n.a. & n.a. & 4 \\
\hline 100.2 & n.a. & n.a. & 5 \\
\hline 100.3 & n.a. & n.a. & 3 \\
\hline 100.4 & n.a. & n.a. & 6 \\
\hline 100.5 & n.a. & n.a. & 3 \\
\hline 100.6 & n.a. & n.a. & 5 \\
\hline avg. & n.a. & n.a. & 4.3 \\
\hline
\end{tabular}


Table 3. one-shot: average expected payoffs of individual sessions

\begin{tabular}{|c|c|c|c|c|c|}
\hline \multirow{2}{*}{ session } & \multicolumn{5}{|c|}{ average expected payoffs (points) } \\
\cline { 2 - 6 } & FP & 1st ANE & 2nd ANE & other NE & sum \\
\hline 46.1 & 0.00 & 1.23 & 0.00 & 14.81 & 16.05 \\
46.2 & 0.00 & 2.47 & 0.00 & 6.17 & 8.64 \\
46.3 & 13.63 & 7.41 & 4.94 & 1.23 & 27.21 \\
46.5 & 1.74 & 3.70 & 0.00 & 16.05 & 21.49 \\
46.6 & 1.14 & 2.47 & 0.00 & 17.28 & 20.89 \\
46.7 & 0.00 & 1.23 & 0.00 & 16.05 & 17.28 \\
46.8 & 1.14 & 0.00 & 0.00 & 6.17 & 7.31 \\
\hline avg. & 1.70 & 1.23 & 0.00 & 13.58 & 16.52 \\
\hline 87.1 & 2.42 & 2.47 & 0.62 & 11.42 & 16.92 \\
87.2 & 4.30 & 7.41 & 0.00 & 6.17 & 17.88 \\
87.3 & 0.00 & 4.94 & 0.00 & 9.88 & 14.81 \\
87.4 & 4.67 & 0.00 & 0.00 & 7.41 & 17.07 \\
87.5 & 6.44 & 7.41 & 2.47 & 3.70 & 17.88 \\
87.6 & 0.00 & 0.94 & 3.70 & 2.47 & 17.56 \\
\hline avg. & 4.12 & 4.12 & 1.03 & 7.20 & 16.46 \\
\hline 99.1 & 1.22 & 2.47 & 0.00 & 14.81 & 18.51 \\
99.2 & 11.00 & 7.41 & 0.00 & 7.41 & 25.81 \\
99.3 & 34.22 & 43.21 & 0.00 & 0.00 & 77.43 \\
99.4 & 11.00 & 3.70 & 0.00 & 6.17 & 20.88 \\
99.5 & 0.00 & 19.75 & 0.00 & 2.47 & 22.22 \\
99.6 & 2.44 & 2.47 & 0.00 & 4.94 & 9.85 \\
\hline avg. & 9.98 & 13.17 & 0.00 & 5.97 & 29.12 \\
\hline 100.1 & n.a. & n.a. & n.a. & 16.05 & 16.05 \\
100.2 & n.a. & n.a. & n.a. & 13.58 & 13.58 \\
100.3 & n.a. & n.a. & n.a. & 13.58 & 13.58 \\
100.4 & n.a. & n.a. & n.a. & 12.35 & 12.35 \\
100.5 & n.a. & n.a. & n.a. & 14.81 & 14.81 \\
100.6 & n.a. & n.a. & n.a. & 14.81 & 14.81 \\
\hline avg. & n.a. & $n . a$. & $n . a$. & 14.20 & 14.20 \\
\hline
\end{tabular}


Table 4. 50 periods: average frequencies of most chosen actions and average expected payoffs in individual "100" sessions

\begin{tabular}{|c|c|c|c|c|c|c|}
\hline \multirow{2}{*}{ session } & \multicolumn{2}{|c|}{ avg. freq. of most chosen action } & \multicolumn{3}{c|}{ avg. expected payoff (points) } \\
\cline { 2 - 7 } & period 1-10 & period 41-50 & period 1-50 & period 1-10 & period 41-50 & period 1-50 \\
\hline 100.1 & 5.0 & 6.4 & 5.6 & 15.56 & 24.82 & 20.35 \\
100.2 & 4.5 & 5.2 & 4.9 & 12.96 & 16.54 & 15.04 \\
100.3 & 4.8 & 4.7 & 4.9 & 12.47 & 16.54 & 14.86 \\
100.4 & 4.6 & 4.5 & 4.5 & 13.21 & 14.44 & 14.22 \\
100.5 & 4.6 & 4.5 & 4.5 & 15.93 & 15.31 & 15.41 \\
100.6 & 4.7 & 5.8 & 4.9 & 10.37 & 20.86 & 14.25 \\
\hline avg. & 4.7 & 5.2 & 4.9 & 13.42 & 18.09 & 15.69 \\
\hline
\end{tabular}


Table 5. 50 periods: average frequencies of actions in individual "46", "87" and "99" sessions

\begin{tabular}{|c|c|c|c|c|c|c|c|c|c|}
\hline \multirow[b]{3}{*}{ session } & \multicolumn{9}{|c|}{ average frequencies } \\
\hline & \multicolumn{3}{|c|}{ focal choices } & \multicolumn{3}{|c|}{ associated actions } & \multicolumn{3}{|c|}{ most chosen other action } \\
\hline & period 1-10 & \begin{tabular}{|l|} 
period 41-50 \\
\end{tabular} & period 1-50 & period 1-10 & period 41-50 & period 1-50 & period 1-10 & period 41-50 & period 1-50 \\
\hline 46.1 & 0.6 & 0.8 & 1.1 & 0.9 & 0.3 & 0.7 & 5.1 & 6.8 & 5.7 \\
\hline 46.2 & 3.1 & 4.0 & 3.4 & 0.3 & 1.7 & 1.3 & 3.8 & 3.7 & 3.9 \\
\hline 46.3 & 8.1 & 7.3 & 7.8 & 2.9 & 5.8 & 4.3 & 2.9 & 2.0 & 2.7 \\
\hline 46.4 & 7.3 & 8.5 & 8.6 & 1.5 & 8.9 & 6.0 & 3.4 & 0.6 & 1.8 \\
\hline 46.5 & 2.8 & 1.2 & 1.9 & 0.9 & 0.5 & 0.8 & 4.0 & 4.3 & 4.1 \\
\hline 46.6 & 1.7 & 0.7 & 1.0 & 0.9 & 0.5 & 1.0 & 4.4 & 4.5 & 4.4 \\
\hline 46.7 & 4.6 & 7.0 & 6.2 & 1.9 & 4.8 & 3.5 & 3.9 & 3.9 & 3.8 \\
\hline 46.8 & 4.1 & 7.3 & 5.6 & 1.2 & 5.3 & 3.9 & 3.9 & 2.6 & 3.2 \\
\hline avg. & 4.0 & 4.6 & 4.5 & 1.3 & 3.5 & 2.7 & 3.9 & 3.6 & 3.7 \\
\hline 87.1 & 6.2 & 10.2 & 9.2 & 1.8 & 4.3 & 3.3 & 3.6 & 2.3 & 2.6 \\
\hline 87.2 & 5.5 & 8.9 & 8.0 & 0.7 & 6.5 & 3.9 & 3.8 & 2.0 & 2.5 \\
\hline 87.3 & 6.6 & 9.5 & 8.1 & 3.9 & 5.5 & 4.9 & 2.9 & 3.0 & 2.9 \\
\hline 87.4 & 5.5 & 9.4 & 8.2 & 2.5 & 4.9 & 3.5 & 3.6 & 2.2 & 2.6 \\
\hline 87.5 & 8.5 & 8.4 & 8.5 & 0.4 & 4.3 & 3.1 & 3.0 & 3.1 & 2.8 \\
\hline 87.6 & 3.9 & 3.0 & 3.1 & 0.3 & 1.9 & 1.1 & 4.3 & 4.3 & 4.5 \\
\hline avg. & 6.0 & 8.2 & 7.5 & 1.6 & 4.6 & 3.3 & 3.5 & 2.8 & 3.0 \\
\hline 99.1 & 6.1 & 13.4 & 9.4 & 0.4 & 0.2 & 0.5 & 3.8 & 2.1 & 3.1 \\
\hline 99.2 & 8.5 & 12.4 & 11.4 & 1.4 & 4.6 & 2.9 & 3.0 & 0.9 & 1.8 \\
\hline 99.3 & 13.1 & 11.5 & 11.7 & 3.1 & 5.4 & 5.1 & 1.3 & 1.0 & 1.0 \\
\hline 99.4 & 7.8 & 11.2 & 10.2 & 1.1 & 3.8 & 2.9 & 3.0 & 2.1 & 2.4 \\
\hline 99.5 & 7.8 & 11.7 & 9.4 & 0.7 & 1.0 & 1.3 & 3.4 & 2.2 & 2.7 \\
\hline 99.6 & 3.7 & 8.5 & 6.3 & 0.8 & 1.4 & 1.7 & 4.6 & 2.9 & 3.5 \\
\hline avg. & 7.8 & 11.5 & 9.7 & 1.3 & 2.7 & 2.4 & 3.2 & 1.9 & 2.4 \\
\hline
\end{tabular}


Table 6. 50 periods: average expected payoffs in individual "46", "87" and "99" sessions

\begin{tabular}{|c|c|c|c|c|c|c|c|c|c|c|c|c|c|c|c|}
\hline \multirow[b]{3}{*}{ session } & \multicolumn{15}{|c|}{ average expected payoffs (points) } \\
\hline & \multicolumn{3}{|c|}{$\overline{F P}$} & \multicolumn{3}{|c|}{ 1st ANE } & \multicolumn{3}{|c|}{ 2nd ANE } & \multicolumn{3}{|c|}{ other NE } & \multicolumn{3}{|c|}{ total } \\
\hline & per. 1-10 & per. 41-50 & per. 1-50 & per. 1-10 & per. $41-50$ & per. $1-50$ & per. 1-10 & per. 41-50 & per. 1-50 & per. 1-10 & per. $41-50$ & per. $1-50$ & per. 1-10 & per. 41-50 & per. 1-50 \\
\hline 46.1 & 0.00 & 0.06 & 0.16 & 0.25 & 0.00 & 0.25 & 0.12 & 0.25 & 0.20 & 14.69 & 27.04 & 20.47 & 15.06 & 27.34 & 21.07 \\
\hline 46.2 & 1.31 & 2.27 & 1.60 & 0.00 & 3.33 & 1.98 & 0.49 & 0.86 & 0.90 & 12.59 & 9.01 & 10.40 & 14.39 & 15.48 & 14.88 \\
\hline 46.3 & 8.86 & 7.55 & 8.25 & 7.41 & 18.15 & 10.17 & 7.16 & 7.66 & 10.12 & 3.33 & 4.57 & 3.33 & 26.76 & 37.92 & 31.88 \\
\hline 46.4 & 7.77 & 6.38 & 9.11 & 0.37 & 62.35 & 34.07 & 8.03 & 3.21 & 6.57 & 5.43 & 2.59 & 2.49 & 21.60 & 74.53 & 52.25 \\
\hline 46.5 & 0.34 & 0.00 & 0.38 & 2.96 & 0.37 & 1.26 & 0.00 & 0.00 & 0.12 & 7.66 & 15.56 & 11.56 & 10.96 & 15.93 & 13.31 \\
\hline 46.6 & 0.11 & 0.17 & 0.09 & 0.86 & 0.25 & 0.39 & 0.00 & 0.12 & 0.15 & 10.62 & 13.70 & 14.17 & 11.60 & 14.24 & 14.81 \\
\hline 46.7 & 2.27 & 3.98 & 4.71 & 5.19 & 28.64 & 13.46 & 0.86 & 1.23 & 3.16 & 8.27 & 7.04 & 7.31 & 16.59 & 40.89 & 28.64 \\
\hline 46.8 & 2.27 & 7.44 & 4.25 & 1.85 & 11.61 & 10.17 & 1.24 & 12.35 & 5.33 & 9.14 & 1.73 & 5.21 & 14.49 & 33.12 & 24.96 \\
\hline avg. & 2.87 & 3.48 & 3.57 & 2.36 & 15.59 & 8.97 & 2.24 & 3.21 & 3.32 & 8.97 & 10.16 & 9.37 & 16.43 & 32.43 & 25.23 \\
\hline 87.1 & 9.99 & 27.92 & 23.59 & 5.43 & 14.69 & 10.47 & 1.60 & 11.98 & 9.58 & 5.43 & 0.25 & 2.10 & 22.46 & 54.84 & 45.74 \\
\hline 87.2 & 8.49 & 14.29 & 15.17 & 1.24 & 52.71 & 26.17 & 0.86 & 0.99 & 2.00 & 7.28 & 6.30 & 4.32 & 17.87 & 74.29 & 47.66 \\
\hline 87.3 & 11.71 & 22.88 & 17.23 & 13.09 & 31.98 & 22.74 & 4.44 & 4.82 & 4.44 & 5.18 & 10.87 & 8.82 & 34.42 & 70.53 & 53.23 \\
\hline 87.4 & 8.70 & 18.05 & 16.13 & 6.05 & 38.89 & 21.19 & 2.59 & 1.23 & 2.07 & 7.41 & 0.86 & 3.33 & 24.75 & 59.03 & 42.73 \\
\hline 87.5 & 17.08 & 18.26 & 18.52 & 1.11 & 25.18 & 17.51 & 0.86 & 1.23 & 1.11 & 3.21 & 9.14 & 5.41 & 22.26 & 53.82 & 42.54 \\
\hline 87.6 & 3.33 & 2.36 & 2.49 & 0.37 & 2.22 & 1.06 & 0.37 & 0.99 & 0.74 & 9.51 & 12.96 & 11.23 & 13.58 & 18.54 & 15.53 \\
\hline avg. & 9.88 & 17.29 & 15.52 & 4.55 & 27.61 & 16.52 & 1.79 & 3.54 & 3.33 & 6.34 & 6.73 & 5.87 & 22.56 & 55.18 & 41.24 \\
\hline 99.1 & 10.63 & 54.27 & 27.77 & 1.23 & 0.74 & 2.22 & 0.12 & 0.99 & 0.81 & 5.68 & 1.23 & 3.60 & 17.67 & 57.23 & 34.41 \\
\hline 99.2 & 22.12 & 46.32 & 39.82 & 8.02 & 21.23 & 16.27 & 0.00 & 14.32 & 6.12 & 3.58 & 0.49 & 1.33 & 33.73 & 82.37 & 63.55 \\
\hline 99.3 & 52.07 & 34.83 & 39.21 & 22.84 & 52.59 & 40.81 & 3.33 & 0.00 & 3.61 & 0.12 & 0.74 & 0.17 & 78.36 & 88.16 & 83.80 \\
\hline 99.4 & 16.87 & 35.20 & 29.85 & 4.94 & 33.58 & 22.03 & 1.11 & 0.00 & 1.16 & 4.07 & 0.99 & 1.63 & 26.99 & 69.77 & 54.66 \\
\hline 99.5 & 4.89 & 40.21 & 20.88 & 4.45 & 8.64 & 7.28 & 0.00 & 0.00 & 0.99 & 1.73 & 1.73 & 2.15 & 11.06 & 50.58 & 31.29 \\
\hline 99.6 & 3.30 & 22.00 & 12.49 & 0.25 & 4.94 & 5.26 & 2.10 & 2.22 & 2.20 & 9.75 & 3.70 & 5.41 & 15.40 & 32.86 & 25.36 \\
\hline avg. & 18.31 & 38.81 & 28.34 & 6.95 & 20.29 & 15.65 & 1.11 & 2.92 & 2.48 & 4.16 & 1.48 & 2.38 & 30.54 & 63.50 & 48.85 \\
\hline
\end{tabular}


Table 7. 50 periods: relative shares of average expected payoffs for each ANE in individual "46", "87" and "99" sessions

\begin{tabular}{|c|c|c|c|c|c|c|}
\hline \multirow{2}{*}{ session } & \multicolumn{6}{|c|}{ average relative shares in expected payoffs of each ANE } \\
\cline { 2 - 7 } & \multicolumn{2}{|c|}{ period 1-10 } & \multicolumn{2}{c|}{ period 41-50 } & \multicolumn{2}{c|}{ period 1-50 } \\
\cline { 2 - 7 } & 1st ANE & 2nd ANE & 1st ANE & 2nd ANE & 1st ANE & 2nd ANE \\
\hline 46.1 & 0.67 & 0.33 & 0.00 & 1.00 & 0.56 & 0.44 \\
46.2 & 0.00 & 1.00 & 0.79 & 0.21 & 0.69 & 0.31 \\
46.3 & 0.51 & 0.49 & 0.70 & 0.30 & 0.50 & 0.50 \\
46.4 & 0.04 & 0.96 & 0.95 & 0.05 & 0.84 & 0.16 \\
46.5 & 1.00 & 0.00 & 1.00 & 0.00 & 0.91 & 0.09 \\
46.6 & 1.00 & 0.00 & 0.67 & 0.33 & 0.73 & 0.27 \\
46.7 & 0.86 & 0.14 & 0.96 & 0.04 & 0.81 & 0.19 \\
46.8 & 0.60 & 0.40 & 0.48 & 0.52 & 0.66 & 0.34 \\
\hline avg. & 0.51 & 0.49 & 0.83 & 0.17 & 0.73 & 0.27 \\
\hline 87.1 & 0.77 & 0.23 & 0.55 & 0.45 & 0.52 & 0.48 \\
87.2 & 0.59 & 0.41 & 0.98 & 0.02 & 0.93 & 0.07 \\
87.3 & 0.75 & 0.25 & 0.87 & 0.13 & 0.84 & 0.16 \\
87.4 & 0.70 & 0.30 & 0.97 & 0.03 & 0.91 & 0.09 \\
87.5 & 0.56 & 0.44 & 0.95 & 0.05 & 0.94 & 0.06 \\
87.6 & 0.50 & 0.50 & 0.69 & 0.31 & 0.59 & 0.41 \\
\hline avg. & 0.72 & 0.28 & 0.89 & 0.11 & 0.83 & 0.17 \\
\hline 99.1 & 0.91 & 0.09 & 0.43 & 0.57 & 0.73 & 0.27 \\
99.2 & 1.00 & 0.00 & 0.60 & 0.40 & 0.73 & 0.27 \\
99.3 & 0.87 & 0.13 & 1.00 & 0.00 & 0.92 & 0.08 \\
99.4 & 0.82 & 0.18 & 1.00 & 0.00 & 0.95 & 0.05 \\
99.5 & 1.00 & 0.00 & 1.00 & 0.00 & 0.88 & 0.12 \\
99.6 & 0.10 & 0.90 & 0.69 & 0.31 & 0.71 & 0.29 \\
\hline avg. & 0.86 & 0.14 & 0.87 & 0.13 & 0.86 & 0.14 \\
\hline
\end{tabular}

* The average for a treatment is weighted is computed as follows: $\Sigma(1 \mathrm{st}$ ANE) $/ \Sigma(1 \mathrm{st}$ ANE+2nd ANE) 